\title{
WHERE DOES RANDOMNESS LEAD IN SPACETIME?
}

\author{
Ismael BAILleul ${ }^{1}$ AND Albert RAUGi ${ }^{2}$
}

\begin{abstract}
We provide an alternative algebraic and geometric approach to the results of [I. Bailleul, Probab. Theory Related Fields 141 (2008) 283-329] describing the asymptotic behaviour of the relativistic diffusion.
\end{abstract}

Mathematics Subject Classification. 60B99, 60J50,60J45, 83A05.

Received March 31, 2008.

\section{INTRODUCTION}

The object of this article is to answer the following question:

\section{"What happens to an object randomly moving in spacetime?"}

This question received in [4] an analytic answer using stochastic calculus and coupling technique. One can see the present article as an alternative way to these results, enlighting their algebraic and geometric content.

We shall adopt as a model of spacetime the geometric framework of special relativity, where the space of events is the Cartesian product $\mathbb{R} \times \mathbb{R}^{d}$, and where the geometry of space is defined by the quadratic form

$$
q(\xi)=\left(\xi^{0}\right)^{2}-\left(\left(\xi^{1}\right)^{2}+\cdots+\left(\xi^{d}\right)^{2}\right)
$$

if $\xi \in \mathbb{R} \times \mathbb{R}^{d}$ has coordinates $\left(\xi^{0}, \xi^{1}, \ldots, \xi^{d}\right)$ in the canonical basis $\left\{\varepsilon_{0}, \varepsilon_{1}, \ldots, \varepsilon_{d}\right\}$.

Rephrase our interrogation: "What is the asymptotic behaviour of an object moving at random in spacetime, continuously, with a speed less than the speed of light?" This question has different flavours depending on who asks it. The probabilist will ask: "What is the tail $\sigma$-algebra of this process?", and the geometer will ask: "Does the space have a (geometrical) boundary such that the object converges almost surely towards some random point of this boundary, encoding completely its asymptotic behaviour?" Answers to these questions can be given as follows. Write $\left\{\xi_{s}\right\}_{s} \geqslant 0$ the random trajectory; we shall denote by $\left(r_{s}, \theta_{s}\right) \in \mathbb{R}_{+} \times \mathbf{S}^{d-1}$ the (Euclidean) polar coordinates of the point $\xi_{s}$ in the hyperplane $\left\{\xi \in \mathbb{R} \times \mathbb{R}^{d} ; \xi^{0}=\xi_{s}^{0}\right\}$, and by $(\Omega, \mathbb{P})$ the probability space where the process $\left\{\xi_{s}\right\}_{s} \geqslant 0$ is constructed, forgetting to mention the initial point of the trajectory.

Theorem 1.1 (probabilist's answer). $\mathbb{P}$-almost surely,

- $\theta_{s}$ converges towards some random asymptotic direction $\theta_{\infty}$,

- $q\left(\xi_{s}, \varepsilon_{0}+\theta_{\infty}\right)$ converges towards some random quantity $\ell_{\infty}$.

\footnotetext{
Keywords and phrases. Random walks on groups, Poisson boundary, special relativity, causal boundary.

1 Cambridge University; i.bailleul@statslab.cam.ac.uk

2 Université Rennes 1; raugi@univ-rennes1.fr
} 

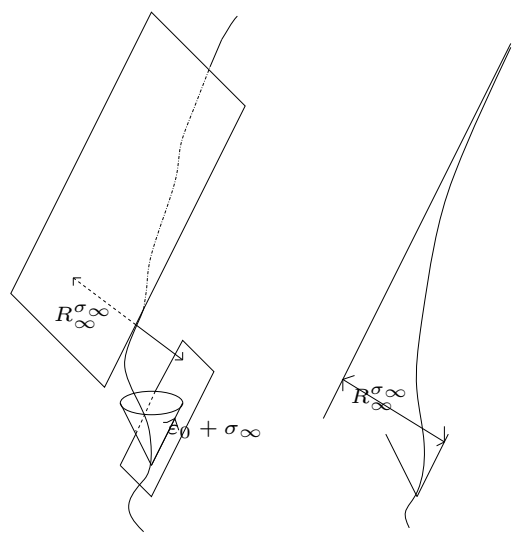

FiguRE 1. Asymptotic behaviour of a typical trajectory.

The $\sigma$-algebra generated by $\theta_{\infty}$ and $\ell_{\infty}$ coincides with the tail $\sigma$-algebra of $\left\{\xi_{s}\right\}_{s \geqslant 0}$ up to $\mathbb{P}$-null sets.

The geometrical meaning of the quantity $\lim _{s \rightarrow+\infty} q\left(\xi_{s}, \varepsilon_{0}+\sigma_{\infty}\right)$ is illustrated in Figure 1: $\xi_{s}$ wanders out to infinity as it approaches the hyperplane parallel to the hyperplane tangent to the null cone in the direction $\varepsilon_{0}+\sigma_{\infty}$, located at a random "height": $\lim _{s \rightarrow+\infty} q\left(\xi_{s}, \varepsilon_{0}+\sigma_{\infty}\right)$.

Theorem 1.2 (geometer's answer). Spacetime $\left(\mathbb{R} \times \mathbb{R}^{d}, q\right)$ has a natural boundary $\mathbf{C}$ such that

- $\left\{\xi_{s}\right\}_{s \geqslant 0}$ converges $\mathbb{P}$-almost surely towards some random point $\xi_{\infty}$ of $\mathbf{C}$,

- the $\sigma$-algebra generated by $\xi_{\infty}$ coincides with the tail $\sigma$-algebra of $\left\{\xi_{s}\right\}_{s} \geqslant 0$ up to $\mathbb{P}$-null sets.

Following Dudley [10], Section 2.1 motivates our model of random motion in spacetime. Section 2.2 explains how one can lift this motion to a motion in the group of affine isometries of $\left(\mathbb{R} \times \mathbb{R}^{d}, q\right)$ and how one can rephrase probabilist's interrogation as a question about random walks on that group. Section 3 explains how Raugi's method, developed in a general setting in [23], provides an explicit description of the invariant $\sigma$-algebra of the random walk; some additional work has to be done to describe its tail $\sigma$-algebra and to rephrase the algebraic result in the form of Theorem 1.1. The use of Raugi's deep and elaborated method in this non trivial simple situation is the occasion to introduce it to the non specialist; with this concern in mind, we have included elementary proofs for some intermediate results that might be well known to specialists. One leaves algebra for geometrical matters in Section 4. There, we address the question of the existence of a boundary to spacetime satisfying geometer's requirements. In the course we prove a theorem which is part of the folklore in Lorentzian geometry but for which one we could not find any reference. Section 5 contains technical proofs which were not included in the preceding sections in order to make them more readable.

We wish to thank the referee for his careful reasing and valuable comments.

\section{From spacetime to Poincaré group}

\subsection{Dudley's model of random motion in spacetime}

As recalled in the introduction, the spacetime of special relativity is the space $\mathbb{R} \times \mathbb{R}^{d}$ equipped with the geometric structure defined by the quadratic form $q$ defined in (1.1); we shall denote it $\mathbb{R}^{1, d}$ in the sequel. An important subset of $\mathbb{R}^{1, d}$ is its upper half-unit sphere

$$
\mathbb{H}=\left\{\xi \in \mathbb{R}^{1, d} ; q(\xi)=1, \xi^{0}>0\right\} .
$$


Although $q$ is not definite, its restriction to any tangent space of $\mathbb{H}$ is definite negative; so, it endows $\mathbb{H}$ with a Riemannian structure. $\mathbb{H}$ is (a model of) the $d$-dimensional hyperbolic space.

One modelizes the motion in spacetime of an object moving at a speed strictly less than the speed of light by a timelike path.

Definition 2.1. A timelike path $\gamma$ in $\mathbb{R}^{1, d}$ is a continuous path $\gamma: I \rightarrow \mathbb{R}^{1, d}, s \mapsto \gamma_{s}=\left(t_{s}, x_{s}\right)$, such that for any $r<s$,

$$
\left|x_{s}-x_{r}\right|<t_{s}-t_{r}
$$

In particular, the time coordinate $t_{r}$ of $\gamma_{r}$ is a continuous increasing function, and $\gamma_{r}$ is absolutely continuous: its left derivative $\dot{\gamma}_{r^{-}}$exists for almost all $r$ and

$$
\gamma_{r}=\gamma_{0}+\int_{0}^{r} \dot{\gamma}_{u^{-}} \mathrm{d} u .
$$

Notation. $S O_{0}(1, d)$ denotes identity's connex component in the linear group of $q$-orthogonal transforms.

- How should one define a random Markovian timelike path started from 0 ? It is first tempting to define the coordinate processes $\gamma \mapsto \gamma_{s}$ on the set $\Gamma$ of all timelike paths $\gamma: \mathbb{R}_{+} \rightarrow \mathbb{R}^{1, d}$ started from 0 and to consider a probability $\mathbb{P}$ on $\Gamma$. Since the laws of physics are supposed to keep the same expression in any pseudo-orthonormal frame of spacetime (Relativity Principle), we require $\mathbb{P}$ to enjoy the same invariance property:

$$
\mathbb{P}\left(\gamma_{t_{1}} \in A_{1}, \ldots, \gamma_{t_{n}} \in A_{n}\right)=\mathbb{P}\left(\gamma_{t_{1}} \in g\left(A_{1}\right), \ldots, \gamma_{t_{n}} \in g\left(A_{n}\right)\right)
$$

for any times $t_{1}<\cdots<t_{n}$, any subsets $A_{1}, \ldots, A_{n}$ of $\mathbb{R}^{1, d}$, and any isometry $g \in S O_{0}(1, d)$. But noting that each timelike path $\gamma$ hits $\mathbb{H}$ in a unique time $T$, the law of the random variable $\gamma_{T}$ should be, under $\mathbb{P}$, a probability measure on $\mathbb{H}$ invariant under the action of $S O_{0}(1, d)$; such a probability does not exists. So, one cannot define an $S O_{0}(1, d)$-invariant probability on the set of timelike paths started from 0 .

This negative result is not surprising since laws of motion in physics always involve position $\gamma_{r}$ in spacetime as well as speed $\dot{\gamma}_{r}$ under the form of a differential equation

$$
\frac{\mathrm{d}\left(\dot{\gamma}_{r}, \gamma_{r}\right)}{\mathrm{d} r}=F\left(\dot{\gamma}_{r}, \gamma_{r}\right)
$$

We shall make the hypothesis that position and speed are recorded in the state space of the process

$$
\mathbb{R}^{1, d} \times\left\{\dot{\xi} \in \mathbb{R}^{1+d} ; q(\dot{\xi})>0, \dot{\xi}^{0}>0\right\}
$$

If one thinks of the process $\left(\gamma_{r}, \dot{\gamma}_{r}\right)$ as modelizing the motion of some particle due to the action of some (continuous) field and to instantaneous collisions with other particles, it is natural to ask $\dot{\gamma}$ to be càdlàg. Noting the Markovian character of the deterministic motion given by (2.1), we shall keep this assumption in the probabilistic model.

\section{Definition 2.2.}

- We define the Poincaré group $\mathcal{G}$ as the group of affine $q$-isometries

$$
\varphi(\zeta)=\mathbf{g}(\zeta)+\xi
$$

with $\xi \in \mathbb{R}^{1, d}$ and a linear part $\mathbf{g}$ in $S O_{0}(1, d)$.

- Writing $(\xi, \mathbf{g})$ for $\varphi$, the group structure of $\mathcal{G}$ is the semi-direct product:

$$
(\xi, \mathbf{g})\left(\xi^{\prime}, \mathbf{g}^{\prime}\right)=\left(\xi+\mathbf{g} \xi^{\prime}, \mathbf{g g}^{\prime}\right)
$$


Given an affine isometry $\mathbf{e}=(\zeta, \mathbf{g})$ of $\mathbb{R}^{1, d}$ and $(\xi, \dot{\xi})$ in the state space, write

$$
\mathbf{e}(\xi, \dot{\xi})=(\mathbf{g} \xi+\zeta, \mathbf{g} \dot{\xi})
$$

the natural action of $\mathbf{e}$ on the state space.

To get a definition of the process $(\gamma, \dot{\gamma})$ in accordance with the Relativity Principle we shall ask its law to be invariant under the action of $\mathcal{G}$. All this leads us to adopt as a model of random timelike path a Markov random process $\left(\gamma_{r}, \dot{\gamma}_{r}\right)$ on $\mathbb{R}^{1, d} \times\left\{\dot{\xi}=(t, x) \in \mathbb{R}^{1+d} ; q(\dot{\xi})>0, t>0\right\}$, defined by a family $\mathbb{P}_{(\xi, \dot{\xi})}$ of probabilities such that for any $(\xi, \dot{\xi})$

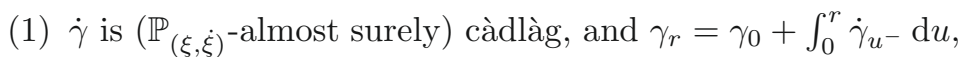

(2) (Invariance) for any affine isometry e of $\mathbb{R}^{1, d}$, any times $t_{1}<\cdots<t_{n}$, and any sets $A_{1}, \ldots, A_{n}$ of the state space

$$
\mathbb{P}_{(\xi, \dot{\xi})}\left(\left(\gamma_{t_{1}}, \dot{\gamma}_{t_{1}}\right) \in A_{1}, \ldots,\left(\gamma_{t_{n}}, \dot{\gamma}_{t_{n}}\right) \in A_{n}\right)=\mathbb{P}_{\mathbf{e}(\xi, \dot{\xi})}\left(\left(\gamma_{t_{1}}, \dot{\gamma}_{t_{1}}\right) \in \mathbf{e} \cdot A_{1}, \ldots,\left(\gamma_{t_{n}}, \dot{\gamma}_{t_{n}}\right) \in \mathbf{e} \cdot A_{n}\right)
$$

Dudley showed in [9] that any such Markov process is a Feller process enjoying the strong Markov property (see Thms. 5.1 and 6.1 of [9]). Note that, as a consequence of the invariance of the law of the process by translations in $\mathbb{R}^{1, d}$, the process $\dot{\gamma}_{r}$ itself is a Markov process.

At this step, it is useful to notice that if one reparameterizes $\gamma$ by the inverse of the previsible additive functional $r \mapsto \int_{0}^{r} \sqrt{q\left(\dot{\gamma}_{u}\right)} \mathrm{d} u$, one obtains a strong Markov process $\left(\underline{\gamma}_{r}, \dot{\gamma}_{r}\right)$ in $\mathbb{R}^{1, d} \times \mathbb{H}$, such that

$\left(1^{\prime}\right) \dot{\gamma}$ is a càdlàg strong Markov process on $\mathbb{H}$, and $\underline{\gamma}_{r}=\underline{\gamma}_{0}+\int_{0}^{r} \underline{\dot{\gamma}}_{u^{-}} \mathrm{d} u$,

$\left(2^{\prime}\right) \overline{\dot{\gamma}}_{u}$ has an $S O_{0}(1, d)$-invariant law.

So we are led to describe the class of càdlàg strong Markov processes on $\mathbb{H}$ with an $S O_{0}(1, d)$-invariant law. One has a clearer understanding of the situation considering $\mathbb{H}$ as the homogeneous space $S O_{0}(1, d) / S O(d)$. Indeed,

Proposition 2.3 ([18], Thm. 2.2, p. 43). There exists a right $S O(d)$-invariant left Lévy process $\left\{g_{u}\right\}$ in $S O_{0}(1, d)$ such that its canonical projection on $\mathbb{H}$ has the same law as $\left\{\dot{\gamma}_{u}\right\}$.

Applebaum and Kunita gave in [3] a characterisation of Lévy processes in Lie groups as solutions of integral equations involving stochastic integrals with respect to a Brownian motion and a Poisson random measure. This description was completed by Applebaum in [2], Theorem 3, p. 396, where he gives a pathwise description of a Lévy process in a Lie group $G$. Defining "by analogy with the Euclidean case a compound Poisson process as the composition of a random number of i.i.d. $G$-valued random variables where the number of terms taken depends on the value of a Poisson process", he employs this compound Poisson process to describe the paths of a Lévy process in $G$ "as the almost sure limit of a sequence of Brownian motions interlaced with random jumps". This characterisation, together with Proposition 2.3, explains Dudley's description of general random Markovian timelike paths: in the same way as Lévy processes in $\mathbb{R}$ are mixings of real Brownian motion and jumps processes, the speed $\dot{\gamma}$ of a Markovian timelike path is a mixing of Brownian trajectories on $\mathbb{H}$ and jump processes (with radial laws) ${ }^{1}$.

Note that the only continuous process of this family of processes is obtained when $\dot{\gamma}$ is a Brownian motion. This uniqueness property is important enough to be recorded in a

Definition 2.4. The relativistic diffusion is the process on $\mathbb{R}^{1, d} \times \mathbb{H}$ obtained as above when $\dot{\gamma}_{s}$ is Brownian motion on $\mathbb{H}$.

\footnotetext{
${ }^{1}$ Note, yet, that Dudley deduced this result from a "static" version of the preceding "dynamic" results, characterizing Fourier transform of some infinitely divisible laws on $\mathbb{H}$, and due to Karpelevich, Schur and Tutubalin [17].
} 


\subsection{From spacetime to Poincaré group}

Relation (2.2) exhibits an action of Poincaré group $\mathcal{G}$ on $\mathbb{R}^{1, d} \times \mathbb{H}$. The object of this section is to describe how one can lift the relativistic diffusion to a diffusion $\left\{\mathbf{e}_{s}\right\}_{s \geqslant 0}$ on $\mathcal{G}$.

a) Brownian motion on $\mathbb{H}$

- Recall $\mathbb{H}$ inherits from $q$ a Riemannian structure. Brownian motion $\left\{\mathbf{g}_{s}^{0}\right\}_{s} \geqslant 0$ on $\mathbb{H}$ is defined as the diffusion with generator half of Laplacian; it has an almost surely infinite lifetime ${ }^{2}$.

Notation. Given $\mathbf{g}^{0} \in \mathbb{H}$, denote by $\mathbb{P}_{\mathbf{g}^{0}}$ the law of Brownian motion $\left\{\mathbf{g}_{s}^{0}\right\}_{s} \geqslant 0$ started from $\mathbf{g}^{0}$.

Polar coordinates $\left(\rho_{s}, \sigma_{s}\right)$ of $\mathbf{g}_{s}^{0}$ satisfy the equations:

$$
\begin{aligned}
& \rho_{s}=\rho_{0}+\frac{d-1}{2} \int_{0}^{s} \operatorname{coth} \rho_{r} \mathrm{~d} r+w_{s}, \\
& \sigma_{s}=\Sigma\left(\int_{0}^{s} \frac{\mathrm{d} r}{\operatorname{sh}^{2} \rho_{r}}\right)
\end{aligned}
$$

where $w$ is a real Brownian motion and $\Sigma$ is an independent Brownian motion on $\mathbb{S}^{d-1}$. We shall only need a few facts about Brownian motion on $\mathbb{H}$; they are collected in the following proposition whose elementary proof uses a comparison theorem on 1-dimensional stochastic differential equations ${ }^{3}$ and the invariance of the law of Brownian motion by the isometric action of $S O_{0}(1, d)$ on $\mathbb{H}$. It is left to the reader.

Proposition 2.5 (asymptotic behaviour of Brownian motion on $\mathbb{H}$ ). Let $\mathbf{g}^{0} \in \mathbb{H}$.

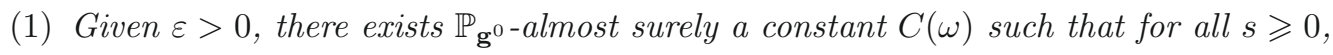

$$
\frac{d-1}{2}(1-\varepsilon) s-C(\omega) \leqslant \rho_{s} \leqslant \frac{d-1}{2}(1+\varepsilon) s+C(\omega)
$$

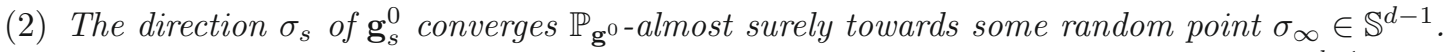

(3) The law of $\sigma_{\infty}$ has a (smooth) density with respect to the uniform probability on $\mathbb{S}^{d-1}$.

Use of half-space coordinates $(y, x) \in \mathbb{R}^{>0} \times \mathbb{R}^{d-1}$ on $\mathbb{H}$ give another insight on that result. The coordinates $\left(y_{s}, x_{s}\right)$ of $\mathbf{g}_{s}^{0}$ satisfy equations

$$
\begin{aligned}
& \mathrm{d} y_{s}=y_{s} \mathrm{~d} w_{s}^{y}-\frac{d}{2} y_{s} \mathrm{~d} s \\
& \mathrm{~d} x_{s}=y_{s} \mathrm{~d} w_{s}^{x}
\end{aligned}
$$

where $\left(w^{y}, w^{x}\right)$ is a $d$-dimensional Brownian motion. One sees on these equations that $y_{s}$ converges exponentially fast to 0 and that $x_{s}$ converges towards some random point of $\mathbb{R}^{d-1}$.

b) A Brownian motion on $\mathbf{S O}_{\mathbf{0}}(\mathbf{1}, \mathbf{d})$

- Following Eells and Elworthy, one usually constructs Brownian motion on an oriented Riemannian manifold $(\mathbb{M}, g)$ of dimension $n$ as the projection of a singular diffusion on the bundle $\mathbb{O M}$ of direct orthonormal frames of TM. Precisely, there exist canonical horizontal vector fields $\left\{H_{i}\right\}_{i=1 \ldots n}$ on $\mathbb{O M}$ such that the differential operator $\sum_{i=1}^{n} H_{i}^{2}$ on $\mathbb{O M}$ induces Laplacian $\triangle^{\mathbb{M}}$ on $\mathbb{M}$, in the sense that if we denote by $\pi$ the natural projection $\mathbb{O M} \rightarrow \mathbb{M}$, we have for all $f \in \mathcal{C}^{2}(\mathbb{M})$,

$$
\left(\sum_{i=1}^{n} H_{i}^{2}\right) f \circ \pi=\left(\triangle^{\mathbb{M}} f\right) \circ \pi
$$

\footnotetext{
${ }^{2}$ Consult [22] or [13], Cor.6.8.

${ }^{3}$ See Theorem 1.1 p. 437 of [16] for example.
} 
These vector fields are defined as follows. Denote by $(x, \mathbf{f})=\left(x,\left(f_{1}, \ldots, f_{n}\right)\right)$ a generic element of $\mathbb{O M}: x \in \mathbb{M}$ and $\mathbf{f}$ is a direct orthonormal basis of $T_{x} \mathbb{M}$. Given $j \in\{1, . ., d\}$, one defines a motion $\left\{\left(x_{s}, \mathbf{f}_{s}\right)\right\}$ in $\mathbb{O M}$ by asking that $\frac{\mathrm{d} x_{s}}{\mathrm{~d} s}=f_{j}(s)$, and $\mathbf{f}$ should be transported parallely along $\left\{x_{s}\right\}$. One defines a vector field $H_{j}$ on $\mathbb{O M}$ looking at the infinitesimal motion of all the points of $\mathbb{O M}$, according to the preceding dynamics. These are the canonical horizontal vector fields.

- In our situation, where $\mathbb{H}$ is the half unit pseudo-sphere of $\mathbb{R}^{1, d}$, the space $\mathbb{O H}$ can be identified with $S O_{0}(1, d)$ and the natural projection is

$$
\mathbf{g}=\left(\mathbf{g}^{0},\left(\mathbf{g}^{1}, \ldots, \mathbf{g}^{d}\right)\right) \in S O_{0}(1, d) \mapsto \mathbf{g}^{0} \in \mathbb{H} .
$$

It is elementary to check that the horizontal vector fields $H_{i}$ are given by the formulas

$$
H_{i}(\mathbf{g})=\mathbf{g} E_{i}, \quad i=1 \ldots d,
$$

where the $E_{i}$ are the matrices $E_{1}=\left(\begin{array}{ccc}0 & 1 & (0) \\ 1 & 0 & \cdots \\ 0 & \vdots & \mathbf{O}_{d-1}\end{array}\right), E_{2}=\left(\begin{array}{cccc}0 & 0 & 1 & (0) \\ 0 & 0 & \cdots \\ 1 & \vdots & & \\ (0) & \vdots & & \mathbf{O}_{d-1}\end{array}\right)$, etc.: $E_{i}$ exchanges $\varepsilon_{0}$ and $\varepsilon_{i}$ and sends the other basis vectors to 0 . These left invariant vector fields give rise to the left invariant differential operator $H_{1}^{2}+\cdots+H_{d}^{2}$ on $S O_{0}(1, d)$. So, if one considers the diffusion $\left\{\mathbf{g}_{s}\right\}_{s \geqslant 0}$ on $S O_{0}(1, d)$ solving the equation

$$
\mathrm{d}_{s}=\mathbf{g}_{s} E_{1} \circ \mathrm{d} w_{s}^{1}+\cdots+\mathbf{g}_{s} E_{d} \circ \mathrm{d} w_{s}^{d},
$$

where the $w^{i}$ s are real independent Brownian motions, the $\mathbb{H}$-valued process $\left\{\mathbf{g}_{s}^{0}\right\}_{s} \geqslant 0$ is by construction a Brownian motion on $\mathbb{H}$.

It is customary in the framework of the study of Lévy processes on Lie groups to call a right-stationary ${ }^{4}$ continuous process with independent right-increments ${ }^{5}$ a right-Brownian motion. The diffusion $\left\{\mathbf{g}_{s}\right\}_{s \geqslant 0}$ is a right-Brownian motion on $S O_{0}(1, d)$. Setting

$$
\xi_{s}=\xi_{0}+\int_{0}^{s} \mathbf{g}_{r}^{0} \mathrm{~d} r
$$

the diffusion $\left\{\left(\xi_{s}, \mathbf{g}_{s}\right)\right\}_{s \geqslant 0}$ in $\mathbb{R}^{1, d} \times S O_{0}(1, d)$ naturally projects to the relativistic diffusion in $\mathbb{R}^{1, d} \times \mathbb{H}$.

c)A Brownian motion and a random walk on $\mathcal{G} \bullet$ The diffusion $\left\{\left(\xi_{s}, \mathbf{g}_{s}\right)\right\}_{s \geqslant 0}$ in $\mathbb{R}^{1, d} \times S O_{0}(1, d)$ is actually a right-Brownian motion on the group $\mathcal{G}$. Indeed, setting $\widetilde{E}_{i}=\left(0, E_{i}\right)$, for $i=1 \ldots d, \widetilde{E}_{0}=\left(\varepsilon_{0}, 0\right)$, and defining the left invariant vector fields $V_{i}$ on $\mathcal{G}$ by the formula

$$
V_{i}((\xi, \mathbf{g}))=(\xi, \mathbf{g}) \widetilde{E}_{i}, i=0 \ldots d,
$$

it is elementary to see that equations (2.6) and (2.7) are equivalent to

$$
\mathrm{d}\left(\left(\xi_{s}, \mathbf{g}_{s}\right)\right)=V_{i}\left(\left(\xi_{s}, \mathbf{g}_{s}\right)\right) \circ \mathrm{d} w_{s}^{i}+V_{0}\left(\left(\xi_{s}, \mathbf{g}_{s}\right)\right) \mathrm{d} s .
$$

Adopting the notation $\mathbf{e}_{s}=\left(\xi_{s}, \mathbf{g}_{s}\right)$ the preceding equation takes its definitive form:

$$
\mathrm{d} \mathbf{e}_{s}=V_{i}\left(\mathbf{e}_{s}\right) \circ \mathrm{d} w_{s}^{i}+V_{0}\left(\mathbf{e}_{s}\right) \mathrm{d} s .
$$

\footnotetext{
${ }^{4}$ For any $s>0$, the process $\left\{\mathbf{g}_{s}^{-1} \mathbf{g}_{s+t}\right\}_{t \geqslant 0}$ has the same law as $\left\{\mathbf{g}_{0}^{-1} \mathbf{g}_{t}\right\}_{t \geqslant 0}$.

${ }^{5}$ For any $s_{1}<\cdots<s_{n}$, the increments $\mathbf{g}_{s_{1}}^{-1} \mathbf{g}_{s_{2}}, \mathbf{g}_{s_{2}}^{-1} \mathbf{g}_{s_{3}}, \ldots, \mathbf{g}_{s_{n-1}}^{-1} \mathbf{g}_{s_{n}}$ are independent.
} 
Using the Markov property and the left invariance of the vector fields $V_{i}$ it is easy to show that $\left\{\mathbf{e}_{s}\right\}_{s \geqslant 0}$ is a right-Brownian motion on $\mathcal{G}^{6}$. Its infinitesimal generator will be denoted by $\widetilde{L}$ :

$$
\widetilde{L}=\frac{1}{2} \sum_{i=1}^{d} V_{i}^{2}+V_{0}
$$

Notice that this operator is not elliptic. Yet this Brownian motion $\left\{\mathbf{e}_{s}\right\}_{s} \geqslant 0$ has useful properties needed in the sequel; they are summarised in the following proposition, proved in Section 5.1.

Notation. Given $\mathbf{e} \in \mathcal{G}$, we shall write $\mathbb{P}_{\mathbf{e}}$ the law of the solution of (2.9) started from $\mathbf{e}$. We write $\operatorname{Haar}(\mathrm{d} \mathbf{a})$ for a Haar measure on $\mathcal{G}^{7}$.

Proposition 2.6.

(1) Smoothness of the law - Given $s>0$, there exists a smooth function $p_{s}$ on $\mathcal{G}$ such that for all bounded Borel function $f$ on $\mathcal{G}$

$$
\mathbb{E}_{\mathbf{e}}\left[f\left(\mathbf{e}_{s}\right)\right]=\int_{\mathcal{G}} p_{s}\left(\mathbf{e}^{-1} \mathbf{a}\right) f(\mathbf{a}) \operatorname{Haar}(\mathrm{d} \mathbf{a}) .
$$

(2) Support of $p_{s}-\operatorname{supp}\left(p_{s}\right)=\left\{\mathbf{e}=(\xi, \mathbf{g}) \in \mathcal{G} ; \frac{\xi}{s} \in \operatorname{ConvHull}(\mathbb{H})\right\}$, where ConvHull( $\left.\mathbb{H}\right)$ is the convex hull of $\mathbb{H} \subset \mathbb{R}^{1, d}$.

(3) Moment - The probability $p_{s}(\mathbf{a})$ Haar $(\mathrm{d} \mathbf{a})$ has a first moment.

We shall recall in Section 3.1 what assertion 3 precisely means. The process $\left\{\mathbf{e}_{s}\right\}_{s} \geqslant 0$ having independent increments, the sequence $\left\{\mathbf{e}_{n}\right\}_{n \geqslant 0}$ is a random walk on $\mathcal{G}$, with jump law $p_{1}(\mathbf{a}) \operatorname{Haar}(\mathrm{da})$. The next section explains how one can reduce the problem of the description of the tail $\sigma$-algebra of the diffusion $\left\{\mathbf{e}_{s}\right\}_{s} \geqslant 0$ to the problem of the description of the invariant $\sigma$-algebra of the random walk $\left\{\mathbf{e}_{n}\right\}_{n} \geqslant 0$.

\subsection{From diffusion to random walk}

Recall that the invariant $\sigma$-algebra $\operatorname{Inv}\left(\mathbf{e}_{s}\right)$ of the diffusion $\left\{\mathbf{e}_{s}\right\}_{s \geqslant 0}$ is generated by the events of the form " $\left\{\mathbf{e}_{s}\right\}_{s \geqslant 0} \in A$ iff $\left\{\mathbf{e}_{s+t}\right\}_{s} \geqslant 0 \in A$ for all $t \geqslant 0$ ". The tail (or asymptotic) $\sigma$-algebra of $\left\{\mathbf{e}_{s}\right\}_{s} \geqslant 0$ is

$$
\operatorname{Tail}\left(\mathbf{e}_{s}\right)=\bigcap_{t \geqslant 0} \sigma\left(\mathbf{e}_{s} ; s \geqslant t\right) .
$$

For general processes one just have $\operatorname{Inv}\left(\mathbf{e}_{s}\right) \subset \operatorname{Tail}\left(\mathbf{e}_{s}\right)$. Besides, the invariant $\sigma$-algebra of the random walk being generated by the events of the form " $\left\{\mathbf{e}_{n}\right\}_{n \geqslant 0} \in A$ iff $\left\{\mathbf{e}_{n+p}\right\}_{n \geqslant 0} \in A$ for all $p \geqslant 0$ ", one just have the a priori inclusions $\operatorname{Inv}\left(\mathbf{e}_{s}\right) \subset \operatorname{Inv}\left(\mathbf{e}_{n}\right) \subset \operatorname{Tail}\left(\mathbf{e}_{s}\right)$. Yet, we shall see that

Theorem 2.7. The two $\sigma$-algebras $\operatorname{Inv}\left(\mathbf{e}_{s}\right)$ and Tail $\left(\mathbf{e}_{s}\right)$ are indistinguishable under any $\mathbb{P}_{\mathbf{e}}$.

As a consequence, the two $\sigma$-algebras $\operatorname{Inv}\left(\mathbf{e}_{s}\right)$ and $\operatorname{Inv}\left(\mathbf{e}_{n}\right)$ coincide up to $\mathbb{P}_{\mathbf{e}}$-null sets for any $\mathbf{e} \in \mathcal{G}$. This brings us back to describe the invariant $\sigma$-algebra of the random walk $\left\{\mathbf{e}_{n}\right\}_{n \geqslant 0}$; this will be done in the forthcoming Section 3. We shall get a result in terms of algebraic quantities; we shall see in Section 3.5 how to interpret them in terms of the relativistic diffusion to recover Theorem 1.1. For the moment we prove Theorem 2.7. Recall an $\widetilde{L}$-harmonic function on $\mathcal{G}$ is a $\mathcal{C}^{2}$ function $h$ such that $\widetilde{L} h=0$. Give a similar definition of a $\left(\partial_{t}+\widetilde{L}\right)$-harmonic function on $\mathbb{R} \times \mathcal{G}$. The proof of Theorem 2.7 relies on the well known correspondence between invariant $\sigma$-algebras and the set of bounded harmonic functions. Note that this correspondence implicitly ${ }^{8}$ uses the hypoellipticity of $\widetilde{L}$ and of its parabolic companion $\partial_{t}+\widetilde{L}$ (an easily verified fact using the explicit formula (2.10) and Hörmander's theorem).

\footnotetext{
${ }^{6}$ See [18] for this fact and a proof that (2.9) has a unique solution.

${ }^{7}$ Notice that $\mathcal{G}$ being a unimodular group, right and left Haar measures coincide.

${ }^{8}$ See Section 9 of [21] for example.
} 


\section{Lemma 2.8.}

- Given any $\operatorname{Inv}\left(\mathbf{e}_{s}\right)$-measurable real bounded random variable $X$, the formula

$$
h(\mathbf{e})=\mathbb{E}_{\mathbf{e}}[X]
$$

defines a bounded $\widetilde{L}$-harmonic function. Any bounded $\widetilde{L}$-harmonic function is of this form.

- Given any Tail $\left(\mathbf{e}_{s}\right)$-measurable real bounded random variable $X$, the formula

$$
h(\mathbf{e})=\mathbb{E}_{\mathbf{e}}[X]
$$

defines a bounded $\left(\partial_{t}+\widetilde{L}\right)$-harmonic function. Any bounded $\left(\partial_{t}+\widetilde{L}\right)$-harmonic function is of this form.

Using this bridge between probability and analysis, Theorem 2.7 can be restated as follows.

Theorem 2.9. Any bounded $\left(\partial_{t}+\widetilde{L}\right)$-harmonic function does not depend on time $t$.

So any bounded $\left(\partial_{t}+\widetilde{L}\right)$-harmonic function is $\widetilde{L}$-harmonic. To prove this theorem, we use the same scheme as in the proof of Corollary 3.2 of [1], but use a Harnack inequality established in [4]. Recall the definition of $p_{r}$ given in Proposition 2.6.

Proof. Let $0<\varepsilon<1$ and $h$ be a bounded $\left(\partial_{t}+\widetilde{L}\right)$-harmonic function. Since the identity

$$
h(t+\varepsilon, \mathbf{e})-h(t, \mathbf{e})=\int\left\{p_{r-\varepsilon}\left(\mathbf{e}^{-1} \mathbf{e}^{\prime}\right)-p_{r}\left(\mathbf{e}^{-1} \mathbf{e}^{\prime}\right)\right\} h\left(t+r, \mathbf{e}^{\prime}\right) \operatorname{Haar}\left(\mathrm{d} \mathbf{e}^{\prime}\right)
$$

holds, for any $\mathbf{e} \in \mathcal{G}, r \geqslant 1$, we have

$$
\begin{aligned}
|h(t+\varepsilon, \mathbf{e})-h(t, \mathbf{e})| & \leqslant\|h\|_{\infty}\left\|p_{r-\varepsilon}\left(\mathbf{e}^{-1} .\right)-p_{r}\left(\mathbf{e}^{-1} \cdot\right)\right\|_{\mathbb{L}^{1}(\text { Haar })} \\
& \leqslant\|h\|_{\infty}\left\|p_{r-\varepsilon}(.)-p_{r}(.)\right\|_{\mathbb{L}^{1}(\text { Haar })} .
\end{aligned}
$$

Now, the function $\left(r, \mathbf{e}^{\prime}\right) \in \mathbb{R}^{>0} \times \mathcal{G} \mapsto p_{r}\left(\mathbf{e}^{\prime}\right)$ being $\left(\partial_{r}-\widetilde{L}^{*}\right)$-harmonic, and this operator being hypoelliptic, one can use the Harnack inequality stated in Theorem 17 in [4]; it ensures the existence of a constant $C$ such that

$$
\forall r \geqslant 1, \forall \mathbf{e}^{\prime} \in \mathcal{G}, \quad p_{r-\varepsilon}\left(\mathbf{e}^{\prime}\right) \leqslant C p_{r}\left(\mathbf{e}^{\prime}\right) .
$$

Inequality (2.12) means that if the probability $p_{r-\varepsilon}(\mathbf{a}) \operatorname{Haar}(\mathrm{d} \mathbf{a})$ puts some mass $m$ on some set, then the probability $p_{r}(\mathbf{a}) \operatorname{Haar}(\mathrm{d} \mathbf{a})$ puts at least some mass $\frac{m}{C}$ on that set. So, the variation distance $\frac{1}{2} \| p_{r-\varepsilon}()-$. $p_{r}(.) \|_{\mathbb{L}^{1}(\text { Haar })}$ between these two probabilities is $\leqslant 1-\frac{1}{C}<1$.

Thus,

$$
\left\|p_{r-\varepsilon}(.)-p_{r}(.)\right\|_{\mathbb{L}^{1}(\text { Haar })} \leqslant 2\left(1-\frac{1}{C}\right)<2 .
$$

Derriennic's $0-2$ law, [8], p. 118, states that one has

$$
\left\|p_{r-\varepsilon}(\mathbf{e}, .)-p_{r}(\mathbf{e}, .)\right\|_{\mathbb{L}^{1}(\text { Haar })} \underset{r \rightarrow+\infty}{\longrightarrow} 0
$$

under the preceding condition; this implies, with (2.11), that

$$
h(t+\varepsilon, .)=h(t, .)
$$

as this holds true for any $\varepsilon>0$, the conclusion follows. 


\section{INVARIANT $\sigma$-ALGEBRA OF THE RANDOM WALK}

As emphasised above, it is the same to determine the invariant $\sigma$-algebra of the random walk $\left\{\mathbf{e}_{n}\right\}_{n \geqslant 0}$ and to determine the set of its bounded harmonic functions $h$ :

$$
\forall \mathbf{e} \in \mathcal{G}, \quad h(\mathbf{e})=\mathbb{E}_{\mathbf{e}}\left[h\left(\mathbf{e}_{1}\right)\right]
$$

We shall concentrate on the description of this set. The method proceeds by finding first what "components" of $\mathbf{e}_{n}$ converge: we shall find three subgroups $\mathcal{D}^{-}, \mathcal{D}^{+}$and $\mathcal{K}$ of $\mathcal{G}$ such that

- the application $\mathcal{D}^{-} \times \mathcal{D}^{+} \times \mathcal{K} \rightarrow \mathcal{G}$ given by $\left(\mathbf{d}^{-}, \mathbf{d}^{+}, \mathbf{k}\right) \mapsto \mathbf{d}^{-} \mathbf{d}^{+} \mathbf{k}$ is a diffeomorphism,

- the $\mathcal{D}^{-}$component $\mathbf{d}_{n}^{-}$of $\mathbf{e}_{n}$ converges almost surely towards some random $\mathbf{d}_{\infty}^{-} \in \mathcal{D}^{-}$.

The paper [4] in some sense studied the random walk $\left\{\mathbf{e}_{n}\right\}_{n \geqslant 0}$ conditioned on $\mathbf{d}_{\infty}^{-}$, using couplings to prove that the invariant $\sigma$-algebra of the conditioned random walk is trivial. Here the algebraic framework enables us to proceed differently and to bring back the problem to show that any $\mathcal{D}^{-}$-left invariant bounded harmonic function is constant; this will be done in Sections 3.3 and 3.4 where Raugi's method is used. In order to make this approach accessible to non specialists we have included elementary proofs for some intermediate results that are perhaps well known to specialists. We first recall in Section 3.1 a few basic facts on measures and bounded harmonic functions on $\mathcal{G}$. The above decomposition of $\mathcal{G}$ is obtained in Section 3.2 and the convergence of the $\mathcal{D}^{-}$component of the random walk in Section 3.3.

\subsection{Basics on measures and bounded harmonic functions}

a) Subadditive function and moments of a measure on $\mathcal{G}$

Definition 3.1. A Borelian function $f: \mathcal{G} \rightarrow \mathbb{R}$ is said to be subadditive if

$$
\forall \mathbf{e}, \mathbf{e}^{\prime} \in \mathcal{G}, \quad f\left(\mathbf{e e}^{\prime}\right) \leqslant f(\mathbf{e})+f\left(\mathbf{e}^{\prime}\right)
$$

The function $f$ is said to be a gauge if there exists a constant $C \in \mathbb{R}$ such that

$$
\forall \mathbf{e}, \mathbf{e}^{\prime} \in \mathcal{G}, \quad f\left(\mathbf{e e}^{\prime}\right) \leqslant f(\mathbf{e})+f\left(\mathbf{e}^{\prime}\right)+C .
$$

The fundamental example of gauge is the gauge associated with a compact neighbourhood $V$ of Id, generating $\mathcal{G}^{9}$ :

$$
\forall \mathbf{e} \in \mathcal{G}, f_{V}(\mathbf{e})=\inf \left\{n \geqslant 1 ; \mathbf{e} \in V^{n}\right\}<\infty .
$$

In our connected group $\mathcal{G}=S O_{0}(1, d) \times \mathbb{R}^{1, d}$, any compact neighbourhood $V$ of Id generates $\mathcal{G}$. So, we drop the assumption " $V$ generates $\mathcal{G}$ " in the sequel. The importance of this example comes from the following fact.

Proposition 3.2 (Guivarch' [14], p. 52). Let $V$ be a compact neighbourhood of Id $\in \mathcal{G}$. For any gauge $f$, there exists a constant $C$, depending on $V$ and $f$, such that

$$
f \leqslant C f_{V}
$$

As a consequence all gauges $f_{V}$ are equivalent: if $V^{\prime}$ is another compact neighbourhood of Id, there exists constants $c\left(V, V^{\prime}\right)$ and $C\left(V, V^{\prime}\right)$ such that

$$
c\left(V, V^{\prime}\right) f_{V} \leqslant f_{V^{\prime}} \leqslant C\left(V, V^{\prime}\right) f_{V}
$$

As a result, if $\mu$ is a non-negative measure on $\mathcal{G}$, and $p \in \mathbb{N}$, the integral $\int f_{V}(\mathbf{e})^{p} \mu(\operatorname{de})$ is finite iff $\int f_{V^{\prime}}(\mathbf{e})^{p} \mu(\operatorname{de})$ is finite.

$$
{ }^{9} \mathcal{G}=\bigcup_{n \in \mathbb{N}} V^{n} .
$$


Definition 3.3. Let $p>0$. A non-negative Borel measure $\mu$ on $\mathcal{G}$ is said to have a moment of order $\boldsymbol{p}$ if

$$
\int f_{V}(\mathbf{e})^{p} \mu(\mathrm{d} \mathbf{e})<\infty
$$

This definition does not depend of the choice of the compact neighbourhood $V$ of the identity.

b) Two properties of bounded harmonic functions

\section{Proposition 3.4.}

1. Any bounded harmonic function $h$ is right uniformly continuous.

2. ([24]) Let $\mathbf{e}_{0} \in \mathcal{G}$, and $h$ be a bounded harmonic function on $\mathcal{G}$. One has $\mathbb{P}_{\mathbf{e}_{0}}$-almost surely, for any $\mathbf{e} \in \operatorname{Supp}\left(p_{1}\right)$

$$
\lim _{n \rightarrow+\infty} h\left(\mathbf{e}_{n} \mathbf{e}\right)=\lim _{n \rightarrow+\infty} h\left(\mathbf{e}_{n}\right)
$$

Proof. 1. Let $\varepsilon>0$ and $\mathcal{V}$ be a compact neighbourhood of $\operatorname{Id} \in \mathcal{G}$. One can find a compact set $K \subset \mathcal{G}$ such that

$$
\forall \widetilde{\mathbf{e}} \in \mathcal{V}, \quad \int_{K^{c}} p_{1}\left(\widetilde{\mathbf{e}}^{-1} \mathbf{e}\right) \operatorname{Haar}(\mathrm{d} \mathbf{e}) \leqslant \varepsilon
$$

Then, for any $\mathbf{e} \in \mathcal{G}$ and $\widetilde{\mathbf{e}} \in \mathcal{V}$, one has

$$
\begin{aligned}
|h(\mathbf{e} \widetilde{\mathbf{e}})-h(\mathbf{e})| & =\left|\int\left\{p_{1}\left((\mathbf{e} \widetilde{\mathbf{e}})^{-1} \mathbf{e}^{\prime}\right)-p_{1}\left(\mathbf{e}^{-1} \mathbf{e}^{\prime}\right)\right\} h\left(\mathbf{e}^{\prime}\right) \operatorname{Haar}\left(\mathrm{d} \mathbf{e}^{\prime}\right)\right| \\
& =\left|\int\left\{p_{1}\left(\widetilde{\mathbf{e}}^{-1} \mathbf{a}\right)-p_{1}(\mathbf{a})\right\} h(\mathbf{a}) \operatorname{Haar}(\mathrm{d} \mathbf{a})\right|=\left|\int_{K} \cdot+\int_{K^{c}} \cdot\right| \\
& \leqslant\left|\int_{K}\left\{p_{1}\left(\widetilde{\mathbf{e}}^{-1} \mathbf{a}\right)-p_{1}(\mathbf{a})\right\} h(\mathbf{a}) \operatorname{Haar}(\mathrm{d} \mathbf{a})\right|+2 \varepsilon\|h\|_{\infty} .
\end{aligned}
$$

Since $p_{1}$ is continuous and $h$ is bounded, one can use dominated convergence theorem in the integral and obtain the existence of a neighbourhood $\widetilde{\mathcal{V}} \subset \mathcal{V}$ of $\mathrm{Id} \in \mathcal{G}$ such that

$$
\forall \mathbf{e} \in \mathcal{G}, \quad \sup _{\widetilde{\mathbf{e}} \in \widetilde{\mathcal{V}}}|h(\mathbf{e} \widetilde{\mathbf{e}})-h(\mathbf{e})| \leqslant 2\|h\|_{\infty} \varepsilon+\varepsilon,
$$

which shows the result.

2. Let $h$ be a bounded harmonic function. Remarking that the inequality

$$
\mathbb{E}_{\mathbf{e}_{0}}\left[\int \sum_{n \geqslant 0}\left(h\left(\mathbf{e}_{n} \mathbf{e}\right)-h\left(\mathbf{e}_{n}\right)\right)^{2} p_{1}(\mathbf{e}) \mathrm{d} \mathbf{e}\right]=\sum_{n \geqslant 0}\left(\mathbb{E}_{\mathbf{e}_{0}}\left[h^{2}\left(\mathbf{e}_{n+1}\right)\right]-\mathbb{E}_{\mathbf{e}_{0}}\left[h^{2}\left(\mathbf{e}_{n}\right)\right]\right)<+\infty,
$$

justifies the $p_{1}(\mathbf{e}) \operatorname{Haar}(\mathrm{d} \mathbf{e}) \otimes \mathbb{P}_{\mathbf{e}_{0}}(\mathrm{~d} \omega)$-almost sure existence of the limit

$$
\lim _{n+\infty}\left(h\left(\mathbf{e}_{n} \mathbf{e}\right)-h\left(\mathbf{e}_{n}\right)\right)=0,
$$

one gets from the $\mathbb{P}_{\mathbf{e}_{0}}$-almost sure convergence of $\left\{h\left(\mathbf{e}_{n}\right)\right\}_{n \geqslant 0}$ the identity:

$$
\lim _{n \rightarrow+\infty} h\left(\mathbf{e}_{n} \mathbf{e}\right)=\lim _{n \rightarrow+\infty} h\left(\mathbf{e}_{n}\right) .
$$

The right uniform continuity of $h$ actually yields the awaited stronger result:

$$
\mathbb{P}_{\mathbf{e}_{0}} \text {-almost surely, for all } \mathbf{e} \in \operatorname{Supp}\left(p_{1}\right), \lim _{n \rightarrow+\infty} h\left(\mathbf{e}_{n} \mathbf{e}\right)=\lim _{n \rightarrow+\infty} h\left(\mathbf{e}_{n}\right) \text {. }
$$




\subsection{Decomposition of $\mathcal{G}$}

One generally studies the behaviour of a random walk on some group $\mathcal{G}$ looking for actions of this group on some space $\mathfrak{X}$ such that for any $x \in \mathfrak{X}$ the trajectory $\left\{\mathbf{e}_{n} \cdot x\right\}_{n \geqslant 0}$ of $x$ converges almost surely; these convergences provide information on $\mathbf{e}_{n}$.

As an example, let us concentrate on the $S O_{0}(1, d)$-part $\mathbf{g}_{n}$ of $\mathbf{e}_{n}$, which is a random walk in its own right. Consider $S O_{0}(1, d)$ as the set of direct isometries of the hyperbolic space, in its half-space representation $\left\{(y, x) \in \mathbb{R}_{+}^{*} \times \mathbb{R}^{d-1}\right\}$. It is an elementary fact that any isometry $\varphi$ of the half-space can uniquely be written

$$
\varphi=t \circ \lambda \circ r
$$

where $t$ is a $\mathbb{R}^{d-1}$-translation, $\lambda$ the homothety $(y, x) \mapsto(\lambda y, \lambda x)$ and $r$ is a hyperbolic rotation with centre $(1,0)$. This decomposition of $\varphi$ is Iwasawa decomposition. Write

$$
\mathbf{g}_{n}=t_{n} \circ \lambda_{n} \circ r_{n}
$$

The random walk $\left\{\mathbf{g}_{n}\right\}_{n \geqslant 0}$ was constructed in Section $\left.2.2, \mathrm{c}\right)$, in such a way that $\mathbf{g}_{n}((1,0))$ is a Brownian motion on $\mathbb{H}$ at time $n$. Since we saw in the remark following Proposition 2.5 that its $\mathbb{R}^{d-1}$ component $x_{n}$ has an almost sure limit, it means that $t_{n}$ converges almost surely. To use the additional information provided by Proposition 2.5 let us re-write Iwasawa decomposition (3.2) in matrix form.

The groups $\mathcal{N}, \mathcal{A}, \mathcal{K}$ of homotheties, translations and rotations have respective Lie algebras

- $\mathfrak{n}$ : the nilpotent algebra

$$
\mathfrak{n}=\left\{\left(\begin{array}{ccc}
0 & 0 & { }^{t} x \\
0 & 0 & { }^{t} x \\
x & -x & \mathbf{0}_{d-1}
\end{array}\right) ; x \in \mathbb{R}^{d-1}\right\}
$$

- $\mathfrak{a}$ : the commutative algebra generated by $\alpha \equiv\left(\begin{array}{ccc}0 & 1 & (0) \\ 1 & 0 & (0) \\ (0) & (0) & \mathbf{0}_{d-1}\end{array}\right){ }^{10}$;

$$
\text { - } \mathfrak{k}=\left\{\left(\begin{array}{ccc}
0 & \ldots & 0 \\
\vdots & s o(d) & \\
0 & &
\end{array}\right)\right\}
$$

In matrix terms the groups $\mathcal{N}, \mathcal{A}, \mathcal{K}$ are described as

Notice that

$$
\begin{gathered}
\mathcal{N}=\operatorname{Exp}(\mathfrak{n})=\left\{N(x)=\left(\begin{array}{ccc}
1+\frac{\|x\|^{2}}{2} & -\frac{\|x\|^{2}}{2} & x^{*} \\
\frac{\|x\|^{2}}{2} & 1-\frac{\|x\|^{2}}{2} & x^{*} \\
x & -x & \mathbf{1}_{d-1}
\end{array}\right) ; x \in \mathbb{R}^{d-1}\right\}, \\
\mathcal{A}=\operatorname{Exp}(\mathfrak{a})=\left\{A(t)=\left(\begin{array}{ccc}
\operatorname{ch} t & \operatorname{sh} t & (0) \\
\operatorname{sh} t & \operatorname{ch} t & (0) \\
(0) & (0) & \mathbf{1}_{d-1}
\end{array}\right) ; t \in \mathbb{R}\right\} \\
\mathcal{K}=\left\{\left(\begin{array}{cc}
1 & (0) \\
(0) & A
\end{array}\right) ; A \in S O(d)\right\}
\end{gathered}
$$

- $\forall x \in \mathbb{R}^{d-1}, N(x)\left(\varepsilon_{0}+\varepsilon_{1}\right)=\varepsilon_{0}+\varepsilon_{1}$, and $N(x)^{*}\left(\varepsilon_{0}-\varepsilon_{1}\right)=\varepsilon_{0}-\varepsilon_{1} ;$

- $\forall t \in \mathbb{R}, A(t)\left(\varepsilon_{0}+\varepsilon_{1}\right)=\mathrm{e}^{t}\left(\varepsilon_{0}+\varepsilon_{1}\right)$, and $A(t)\left(\varepsilon_{0}-\varepsilon_{1}\right)=\mathrm{e}^{-t}\left(\varepsilon_{0}-\varepsilon_{1}\right)$;

\footnotetext{
${ }^{10}$ We denote by $\mathbf{0}_{d-1}$ the $(d-1) \times(d-1)$ zero matrix.
} 
- $\forall x \in \mathbb{R}^{d-1}, \forall t \in \mathbb{R}, A(t) N(x) A^{-1}(t)=N\left(\mathrm{e}^{t} x\right)$;

- $\forall x, y \in \mathbb{R}^{d-1}, N(x) N(y)=N(x+y)$.

Identity (3.2) for $\mathbf{g}_{n}$ takes the matrix form

$$
\mathbf{g}_{n}=N\left(x_{n}\right) A\left(t_{n}\right) K_{n},
$$

with $x_{n} \in \mathbb{R}^{d-1}, t_{n} \in \mathbb{R}$ and $K_{n} \in \mathcal{K}$.

Denote by $\langle.,$.$\rangle the Euclidean scalar product on \mathbb{R}^{1+d}$. On the one hand one has

$$
\left\langle\varepsilon_{0}-\varepsilon_{1}, \mathbf{g}_{s}^{0}\right\rangle=\left\langle\varepsilon_{0}-\varepsilon_{1}, N\left(x_{s}\right) A\left(t_{s}\right) \varepsilon_{0}\right\rangle=\left\langle\varepsilon_{0}-\varepsilon_{1}, A\left(t_{s}\right) \varepsilon_{0}\right\rangle=\mathrm{e}^{-t_{s}} .
$$

On the other hand, using polar coordinates $\left(\rho_{s}, \sigma_{s}\right) \in \mathbb{R}_{+}^{*} \times \mathbb{S}^{d-1}$ of $\mathbf{g}_{s}^{011}: \mathbf{g}_{s}^{0}=\operatorname{ch}\left(\rho_{s}\right) \varepsilon_{0}+\operatorname{sh}\left(\rho_{s}\right) \sigma_{s} \in \mathbb{R}^{1, d}$, one has

$$
\left\langle\varepsilon_{0}-\varepsilon_{1}, \mathbf{g}_{s}^{0}\right\rangle=\operatorname{ch}\left(\rho_{s}\right)-\operatorname{sh}\left(\rho_{s}\right) \sigma_{s}^{1} .
$$

where $\sigma_{s}^{1}=\left\langle\varepsilon_{1}, \sigma_{s}\right\rangle$. Now $\mathbf{g}_{s}^{0}$ being a Brownian motion on $\mathbb{H}$ one knows from Proposition 2.5 that

- $\rho_{s}=\frac{d-1}{2} s+o(s)$;

- $\sigma_{s}^{1}$ converges almost surely towards a (random) limit $\sigma_{\infty}^{1}$ such that $\left|\sigma_{\infty}^{1}\right|<1$;

as a consequence,

$$
\begin{aligned}
\left\langle\varepsilon_{0}-\varepsilon_{1}, \mathbf{g}_{s}^{0}\right\rangle & =\operatorname{ch}\left(\rho_{s}\right)-\operatorname{sh}\left(\rho_{s}\right) \sigma_{s}^{1}=\frac{1-\sigma_{s}^{1}}{2} \mathrm{e}^{\rho_{s}}+o(1) \\
& =\frac{1-\sigma_{s}^{1}}{2} \mathrm{e}^{\frac{d-1}{2} s+o(s)}+o(1)=\mathrm{e}^{-t_{s}} .
\end{aligned}
$$

Proposition 3.5. The component $N\left(x_{n}\right)$ of $\mathbf{g}_{n}$ in Iwasawa decomposition $\mathbf{g}_{n}=N\left(x_{n}\right) A\left(t_{n}\right) K_{n}$ converges almost surely, and $\frac{t_{n}}{n} \underset{n \rightarrow+\infty}{\longrightarrow}-\frac{d-1}{2}$, a.s.

To get information on what happens in the $\mathbb{R}^{1, d}$ component of $\mathbf{e}_{n}$ we shall define an action of some sub-group of $\mathcal{G}$ on a vector space of polynomials on the Lie algebra $\mathfrak{g}$ of $\mathcal{G}$. Before doing so we need a few notations.

Identify $S O_{0}(1, d)$ with a subgroup of $\mathcal{G}$, and $s o(1, d)$ with a sub-algebra of $\mathfrak{g}$. Set

- $\mathfrak{d}=\mathbb{R}^{1+d} \oplus \mathfrak{n} \oplus \mathfrak{a}$,

- $\mathfrak{d}^{-}=\mathbb{R}\left(\varepsilon_{0}+\varepsilon_{1}\right) \oplus \mathfrak{n}$,

- $\mathfrak{d}^{+}=\left(\mathbb{R}\left(\varepsilon_{0}-\varepsilon_{1}\right) \oplus\left\langle\varepsilon_{2}, \ldots, \varepsilon_{d}\right\rangle\right) \oplus \mathfrak{a}^{12}$,

so that

$$
\mathfrak{d}=\mathfrak{d}^{-} \oplus \mathfrak{d}^{+} .
$$

As is clear from equation (2.4), the behaviour of the $y$ component of Brownian motion in the half-space model explains the convergence of its $x$ component. In the algebraic framework, it is the behaviour of $A_{n}$ which explains the dynamics of $\mathbf{g}_{n}$. The preceding decomposition of $\mathfrak{g}$ is adapted to the structure of $\mathcal{A}$ : $\left(\varepsilon_{0}+\varepsilon_{1}\right)$ is contracted by $A_{n},\left(\varepsilon_{0}-\varepsilon_{1}\right)$ is dilated, and $\left\langle\varepsilon_{2}, \ldots, \varepsilon_{d}\right\rangle$ is stable. This decomposition has the following straightforward properties:

- $\mathfrak{d}^{-}$and $\mathfrak{d}^{+}$are Lie sub-algebras of $\mathfrak{g}$, with associated groups

$$
\mathcal{D}^{-} \equiv \mathbb{R}\left(\varepsilon_{0}+\varepsilon_{1}\right) \times \mathcal{N} \text {, and } \mathcal{D}^{+} \equiv\left(\mathbb{R}\left(\varepsilon_{0}-\varepsilon_{1}\right) \oplus\left\langle\varepsilon_{2}, \ldots, \varepsilon_{d}\right\rangle\right) \times \mathcal{A} ;
$$

- $\mathfrak{d}$ is the Lie algebra of the group $\mathcal{D} \equiv \mathbb{R}^{1+d} \times \mathcal{N} \mathcal{A}$; and

- the group $\left(\mathcal{D}^{-},.\right)$is isomorphic to the Abelian group $\left(\mathfrak{d}^{-},+\right)$.

\footnotetext{
${ }^{11}$ We see $\mathbb{S}^{d-1}$ as a subset of $\mathbb{R}^{d} \subset \mathbb{R}^{1, d}$.

${ }^{12}\left\langle\varepsilon_{2}, \ldots, \varepsilon_{d}\right\rangle$ is the vector space spanned by $\varepsilon_{2}, \ldots, \varepsilon_{d}$.
} 
Proposition 3.6 (decomposition of $\mathcal{G}$ ). The application $\mathcal{D}^{-} \times \mathcal{D}^{+} \times \mathcal{K} \rightarrow \mathcal{G}$ given by $\left(\mathbf{d}^{-}, \mathbf{d}^{+}, \mathbf{k}\right) \mapsto \mathbf{d}^{-} \mathbf{d}^{+} \mathbf{k}$ is a diffeomorphism.

Notation. It will be convenient to write

$$
\mathbf{e}=\mathbf{d}^{-} \mathbf{d}^{+} \mathbf{k}=\pi_{\mathcal{D}}(\mathbf{e}) \mathbf{k}=\pi_{\mathcal{D}^{-}}(\mathbf{e}) \mathbf{d}^{+} \mathbf{k},
$$

with $\pi_{\mathcal{D}}(\mathbf{e})=\mathbf{d}^{-} \mathbf{d}^{+} \in \mathcal{D}$ and $\pi_{\mathcal{D}^{-}}(\mathbf{e})=\mathbf{d}^{-} \in \mathcal{D}^{-}$.

\subsection{Convergence on $\mathcal{D}^{-}$}

a) An action of $\mathcal{D}$ on $\mathfrak{d}^{-} \bullet$ The map

$$
\left(\mathbf{d}, \mathbf{d}^{-}\right) \in \mathcal{D} \times \mathcal{D}^{-} \mapsto \mathbf{d} . \mathbf{d}^{-}:=\pi_{\mathcal{D}^{-}}\left(\mathbf{d d}^{-}\right) \in \mathcal{D}^{-}
$$

defines an action of $\mathcal{D}$ on $\mathcal{D}^{-}$which corresponds to the action of $\mathcal{D}$ on the homogeneous space $\mathcal{D} / \mathcal{D}^{+}$, identified with $\mathcal{D}^{-}$. Given any $s, r \in \mathbb{R}, y, x \in \mathbb{R}^{d-1}$ and $\xi \in \mathbb{R}^{d+1}$, we have

$$
(\xi, N(y) A(s))\left(r\left(\varepsilon_{0}+\varepsilon_{1}\right), N(x)\right)=\left(\xi+r \mathrm{e}^{s}\left(\varepsilon_{0}+\varepsilon_{1}\right), N\left(y+\mathrm{e}^{s} x\right) A(s)\right)
$$

which can be written

$$
\left(0, N\left(y+\mathrm{e}^{s} x\right)\right)\left(\left(\left\langle N^{-1}\left(y+\mathrm{e}^{s} x\right) \xi, \varepsilon_{0}+\varepsilon_{1}\right\rangle+r \mathrm{e}^{t}\right)\left(\varepsilon_{0}+\varepsilon_{1}\right), \mathbf{1}_{d+1}\right)(\zeta, A(t))
$$

where $\zeta=N^{-1}\left(y+\mathrm{e}^{s} x\right) \xi-\left\langle N^{-1}\left(y+\mathrm{e}^{s} x\right) \xi, \varepsilon_{0}+\varepsilon_{1}\right\rangle\left(\varepsilon_{0}+\varepsilon_{1}\right) \in \mathbb{R}\left(\varepsilon_{0}-\varepsilon_{1}\right) \oplus\left\langle\varepsilon_{2}, \ldots, \varepsilon_{d}\right\rangle$. Therefore, for $\mathbf{d}=(\xi, N(y) A(s)) \in \mathcal{D}$ and $\mathbf{d}^{-}=\left(r\left(\varepsilon_{0}+\varepsilon_{1}\right), N(x)\right) \in \mathcal{D}^{-}$,

$$
\text { d.d } \mathbf{d}^{-}=\left(\left(\left\langle N^{-1}\left(y+\mathrm{e}^{s} x\right) \xi, \varepsilon_{0}+\varepsilon_{1}\right\rangle+r \mathrm{e}^{s}\right)\left(\varepsilon_{0}+\varepsilon_{1}\right), N\left(y+\mathrm{e}^{s} x\right)\right) .
$$

Now, via the exponential map, we identify the Abelian Lie group $\mathcal{N}$ with its Lie algebra $\mathfrak{n}$, itself identified with $\mathbb{R}^{d-1}$. This identification gives us the following action of $\mathcal{D}$ on $\mathfrak{d}^{-}$, identified to $\mathbb{R} \times \mathbb{R}^{d-1}$ :

$$
\mathbf{d}(r, x)=\left(\left\langle N^{-1}\left(y+\mathrm{e}^{s} x\right) \xi, \varepsilon_{0}+\varepsilon_{1}\right\rangle+r \mathrm{e}^{s}, y+\mathrm{e}^{s} x\right) .
$$

If one decomposes $\mathbf{d}=\left(t\left(\varepsilon_{0}+\varepsilon_{1}\right), N(y)\right)(\zeta, A(s)) \in \mathcal{D}$ into its $\mathcal{D}^{-}$-part and its $\mathcal{D}^{+}$-part, then one reads on the preceding formula that

$$
\mathbf{d}(r, x)=\left(t+\mathrm{e}^{s} r+\left\langle N\left(-\mathrm{e}^{s} x\right) \zeta, \varepsilon_{0}+\varepsilon_{1}\right\rangle, \mathrm{e}^{s} x+y\right) .
$$

As is clear, $\mathcal{D}$ do not have an affine action on $\mathfrak{d}^{-}$; nonetheless we shall see that this action induces a linear action on some space of polynomials on $\mathfrak{d}^{-}$.

b) Polynomials on $\mathfrak{d}^{-} \bullet$ The algebra $\mathfrak{d}^{-}$is a nilpotent algebra of height 2 :

$$
\left[\mathfrak{d}^{-}, \mathfrak{d}^{-}\right]=\mathbb{R}\left(\varepsilon_{0}+\varepsilon_{1}, 0\right), \quad\left[\mathfrak{d}^{-}, \mathbb{R}\left(\varepsilon_{0}+\varepsilon_{1}, 0\right)\right]=0 .
$$

As such, it has a natural graded structure. We consider the algebra of polynomial functions $\mathbb{R}\left(x_{1}, \ldots, x_{d-1}, r\right)$ on $\mathfrak{d}^{-}$, provided with the adapted concept of degree:

$$
\begin{aligned}
& d^{\circ} x_{i}=1, \quad \forall i=1 \ldots d-1, \\
& d^{\circ} r=2 .
\end{aligned}
$$


c) An action of $\mathcal{D}$ on polynomials on $\mathfrak{d}^{-}$, identified to $\mathbb{R} \times \mathbb{R}^{d-1} \bullet$ The group $\mathcal{D}$ acts linearly on $\mathbb{R}\left(x_{1}, \ldots, x_{d-1}, r\right)$ in the following way

$$
\forall P \in \mathbb{R}\left(x_{1}, \ldots, x_{d-1}, r\right), \forall \mathbf{d} \in \mathcal{D}, \quad(P . \mathbf{d})(r, x)=P(\mathbf{d} .(r, x)) .
$$

Moreover, as $N(x)$ is a matrix with quadratic coefficients in $x$, one reads on formula (3.4) that this action leaves the vectorial sub-space generated by the family $\left\{1 ; x_{1}, \ldots, x_{d-1} ;\left(x_{1}\right)^{2}, \ldots,\left(x_{d-1}\right)^{2}, r\right\}$ invariant. The matrix of the linear action of $\mathbf{d}=(\xi, N(y) A(s)) \in \mathcal{D}$ in the basis $\left\{\mathbf{1} ; x_{1}, \ldots, x_{d-1} ;\left(x_{1}\right)^{2}, \ldots,\left(x_{d-1}\right)^{2}, r\right\}$ is

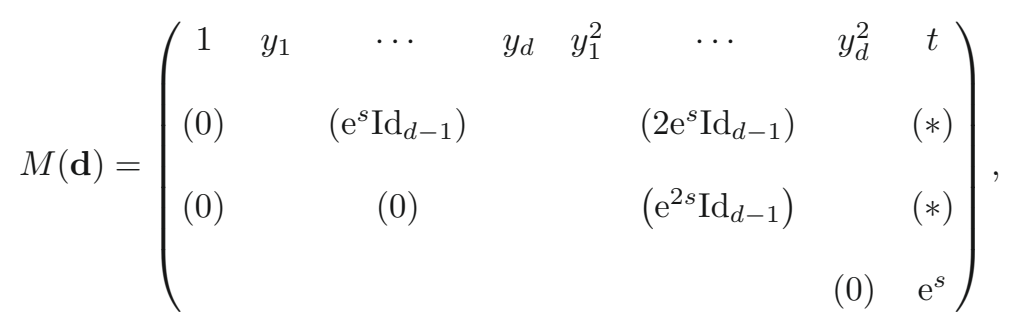

where $(*)$ depends on $\mathbf{d}$. We shall write it in a more concise form

$$
M(\mathbf{d})=\left(\begin{array}{cc}
1 & U(\mathbf{d}) \\
(0) & T(\mathbf{d})
\end{array}\right)=\left(\begin{array}{ccc}
1 & \bar{U}(\mathbf{d}) & t \\
(0) & \bar{T}(\mathbf{d}) & V(\mathbf{d}) \\
0 & (0) & \mathrm{e}^{s}
\end{array}\right) .
$$

d) Convergence of the $\mathcal{D}^{-}$-component $\bullet$ Let now see what this action of $\mathcal{D}$ on polynomials of degree $\leqslant 2$ tells us about $\mathbf{e}_{n}$. We shall write $\mathbf{e}_{n}=\mathbf{d}_{n} \mathbf{k}_{n}$, with $\mathbf{d}_{n} \in \mathcal{D}$ and $\mathbf{k}_{n} \in \mathcal{K}$. For convenience, denote by $\mu$ the jump law of $\mathbf{e}_{n}$, and write

$$
\mathbf{e}_{n+1}=\mathbf{e}_{n} \mathbf{y}_{n+1},
$$

where the $\mathbf{y}_{i}$ are independent random variables with common law $\mu$. As

$$
\mathbf{e}_{n+1}=\mathbf{d}_{n+1} \mathbf{k}_{n+1}=\mathbf{e}_{n} \mathbf{y}_{n+1}=\mathbf{d}_{n} \mathbf{k}_{n} \mathbf{y}_{n+1}=\mathbf{d}_{n} \pi_{\mathcal{D}}\left(\mathbf{k}_{n} \mathbf{y}_{n+1}\right) \pi_{\mathcal{K}}\left(\mathbf{k}_{n} \mathbf{y}_{n+1}\right),
$$

we have

$$
\mathbf{d}_{n+1}=\pi_{\mathcal{D}}\left(\mathbf{e}_{0} \mathbf{y}_{1}\right) \pi_{\mathcal{D}}\left(\mathbf{k}_{1} \mathbf{y}_{2}\right) \ldots \pi_{\mathcal{D}}\left(\mathbf{k}_{n} \mathbf{y}_{n+1}\right)
$$

and $^{13}$

$$
M\left(\mathbf{d}_{n}\right)=M\left(\pi_{\mathcal{D}}\left(\mathbf{k}_{n-1} \mathbf{y}_{n}\right)\right) M\left(\pi_{\mathcal{D}}\left(\mathbf{k}_{n-2} \mathbf{y}_{n-1}\right)\right) \cdots M\left(\pi_{\mathcal{D}}\left(\mathbf{e}_{0} \mathbf{y}_{1}\right)\right) .
$$

To prove the $\mathbb{P}_{\mathbf{e}_{0}}$-almost sure convergence of the $\mathcal{D}^{-}$-component of the random walk $\left\{\mathbf{e}_{n}\right\}_{n \geqslant 0}$ we prove the convergence of the matrices $M\left(\mathbf{d}_{n}\right)$.

Setting, with the convention $\mathbf{k}_{0}=\mathbf{e}_{O}$,

$$
M\left(\pi_{\mathcal{D}}\left(\mathbf{k}_{p} \mathbf{y}_{p+1}\right)\right)=\left(\begin{array}{cc}
1 & U_{p} \\
(0) & T_{p}
\end{array}\right)
$$

we have

$$
U\left(\mathbf{d}_{n}\right)=U_{0}+U_{1} T_{0}+U_{2} T_{1} T_{0}+\ldots+U_{n-1} T_{n-2} \cdots T_{0},
$$

and

$$
T_{n-1} \cdots T_{0}=T\left(\mathbf{d}_{n}\right) .
$$

We temporarily admit the following lemma, in which $\|\cdot\|$ is any norm on matrices.

Lemma 3.7. One has $\mathbb{P}_{\mathbf{e}_{0}}$-almost surely

\footnotetext{
${ }^{13}$ Remember we have a right action.
} 
(1) $\varlimsup\left\|M\left(\pi_{\mathcal{D}}\left(\mathbf{k}_{n-1} \mathbf{y}_{p}\right)\right)\right\|^{1 / n} \leqslant 1$,

(2) $\lim \left\|T\left(\mathbf{d}_{n}\right)\right\|^{\frac{1}{n}}=\mathrm{e}^{-\frac{d-1}{2}}<1$.

It follows from this lemma that $\varlimsup$ lim $\left\|U_{n}\right\|^{\frac{1}{n}} \leqslant \varlimsup\left\|M\left(\pi_{\mathcal{D}}\left(\mathbf{k}_{n-1} \mathbf{y}_{n}\right)\right)\right\|^{\frac{1}{n}} \leqslant 1$; we thus see that the right hand side of (3.7) converges as $n \rightarrow+\infty$. Together with the first point of the Lemma 3.7, this proves the convergence of $M\left(\mathbf{d}_{n}\right)$.

Theorem 3.8 (convergence theorem). The $\mathcal{D}^{-}$-component $\mathbf{d}_{n}^{-}$of $\mathbf{e}_{n}$ converges $\mathbb{P}_{\mathbf{e}_{0}}$-almost surely, for every $\mathbf{e}_{0} \in \mathcal{G}$.

It remains to prove Lemma 3.7. All norms being equivalent, it does not matter which norm we use; we choose to work with an algebra norm, for which $\|A B\| \leqslant\|A\|\|B\|$, for any matrices $A, B$.

Proof. 1. Set for $\mathbf{e} \in \mathcal{G}$

$$
\phi(\mathbf{e}) \equiv \sup _{\mathbf{k} \in \mathcal{K}} \log \left\|M\left(\pi_{\mathcal{D}}(\mathbf{k e})\right)\right\|
$$

$\mathcal{K}$ being compact, the supremum is finite.

Lemma 3.9. $\phi$ is subadditive: for any $\mathbf{e}, \mathbf{e}^{\prime} \in \mathcal{G}, \phi\left(\mathbf{e e}^{\prime}\right) \leqslant \phi(\mathbf{e})+\phi\left(\mathbf{e}^{\prime}\right)$.

Proof of Lemma 3.9. The inequality is a direct consequence of the identity

$\forall \mathbf{k} \in \mathcal{K}, \mathbf{e}, \mathbf{e}^{\prime} \in \mathcal{G}, \quad \pi_{\mathcal{D}}\left(\mathbf{k e e}^{\prime}\right)=\pi_{\mathcal{D}}\left(\pi_{\mathcal{D}}(\mathbf{k e}) \pi_{\mathcal{K}}(\mathbf{k e}) \mathbf{e}^{\prime}\right)=\pi_{\mathcal{D}}(\mathbf{k e}) \pi_{\mathcal{D}}\left(\pi_{\mathcal{K}}(\mathbf{k e}) \mathbf{e}^{\prime}\right)$.

It follows from Proposition 3.2 that $\phi$ is bounded by a multiple of a gauge, and that since $\mu$ has a first moment (and $\phi \geqslant 0$ ) one has

$$
\int \phi(\mathbf{e}) \mu(\mathrm{d} \mathbf{e})<\infty
$$

Therefore,

$$
\sum_{n \geqslant 1} \mu(\phi \geqslant n c)<\infty, \text { for any } c>0
$$

and

$$
\sum_{n \geqslant 2} \mathbb{P}_{\mathbf{e}_{0}}\left(\log \left\|M\left(\pi_{\mathcal{D}}\left(\mathbf{k}_{n-1} \mathbf{y}_{n}\right)\right)\right\| \geqslant n c\right) \leqslant \sum_{n \geqslant 2} \mathbb{P}\left(\phi\left(\mathbf{y}_{n}\right) \geqslant n c\right)=\sum_{n \geqslant 2} \mu(\phi \geqslant n c)<\infty .
$$

An application of Borel-Cantelli lemma yields the $\mathbb{P}_{\mathbf{e}_{0}}$-almost sure inequality

$$
\varlimsup \varlimsup_{\lim }\left\|M\left(\pi_{\mathcal{D}}\left(\mathbf{k}_{n-1} \mathbf{y}_{n}\right)\right)\right\|^{\frac{1}{n}} \leqslant \mathrm{e}^{c} .
$$

As this inequality holds for any $c>0$, one has $\mathbb{P}_{\mathbf{e}_{0}}$-almost surely

$$
\varlimsup\left\|M\left(\pi_{\mathcal{D}}\left(\mathbf{k}_{n-1} \mathbf{y}_{n}\right)\right)\right\|^{\frac{1}{n}} \leqslant 1 .
$$

2. Write

$$
M\left(\pi_{\mathbf{d}}\left(\mathbf{k}_{n} \mathbf{y}_{n}\right)\right)=\left(\begin{array}{cc}
1 & \mathbf{u}_{n} \\
(0) & \mathbf{t}_{n}
\end{array}\right)=\left(\begin{array}{ccc}
1 & \overline{\mathbf{u}}_{n} & \rho_{n} \\
(0) & \bar{\tau}_{n} & \overline{\mathbf{v}}_{n} \\
0 & (0) & \mathrm{e}^{u_{n}}
\end{array}\right)
$$

using obvious notations. One sees from relation (3.6) that

$$
\begin{aligned}
V\left(\mathbf{d}_{n}\right) & =\sum_{k=0}^{n-1} \bar{\tau}_{n-1} \cdots \bar{\tau}_{k+1} \overline{\mathbf{v}}_{k} \mathrm{e}^{u_{k-1}+\cdots+u_{0}}=\sum_{k=0}^{n-1} \bar{T}\left(\mathbf{d}_{n}\right) \bar{T}\left(\mathbf{d}_{k+1}\right)^{-1} \overline{\mathbf{v}}_{k} \mathrm{e}^{s_{k}} \\
& =\bar{T}\left(\mathbf{d}_{n}\right) \sum_{k=0}^{n-1} \mathrm{e}^{s_{k+1}} \bar{T}\left(\mathbf{d}_{k+1}\right)^{-1} \mathrm{e}^{-u_{k}} \overline{\mathbf{v}}_{k}
\end{aligned}
$$


As one has $\lim _{n,+\infty}\left\|\mathrm{e}^{s_{k}} \bar{T}\left(\mathbf{d}_{k}\right)^{-1}\right\|^{\frac{1}{n}}=1$ and $\varlimsup\left\|\mathrm{e}^{-u_{k}} \overline{\mathbf{v}}_{k}\right\|^{\frac{1}{n}} \leqslant 1$, from the first point of the lemma, one deduces easily that

and, therefore, that $\lim \left\|T\left(\mathbf{d}_{n}\right)\right\|^{\frac{1}{n}}=\mathrm{e}^{-\frac{d-1}{2}}$.

$$
\varlimsup=V\left(\mathbf{d}_{n}\right) \|^{\frac{1}{n}} \leqslant \mathrm{e}^{-\frac{d-1}{2}},
$$

We are now ready to determine the invariant $\sigma$-algebra of $\left\{\mathbf{e}_{n}\right\}_{n \geqslant 0}$. As explained earlier, it is equivalent to determine the set of its $\mu$-bounded harmonic functions.

\subsection{Invariant $\sigma$-algebra of the random walk}

The description of the set of $\mu$-bounded harmonic functions, leads us to introduce the space of the jumps $\left\{\mathbf{y}_{n}=\mathbf{e}_{n-1}^{-1} \mathbf{e}_{n}\right\}_{n \in \mathbb{N}^{*}}$ of the trajectories, which define a sequence of independent random variables with common law $\mu$, instead of the trajectories $\left\{\mathbf{e}_{n}\right\}_{n \in \mathbb{N}}$. In other words, we consider the product space $\widetilde{\Omega}=\mathcal{G}^{\mathbb{N}^{*}}$, provided with its Borel $\sigma$-algebra and the product measure $\widetilde{\mathbb{P}}=\otimes_{\mathbb{N}^{*}} \mu$. Under $\widetilde{\mathbb{P}}$, the coordinate maps $\left\{Y_{n}\right\}_{n \geqslant 1}$ of $\widetilde{\Omega}$ are independent, with common law $\mu$. We call $\theta$ the shift on $\widetilde{\Omega}$ : for any $\omega \in \widetilde{\Omega}$,

$$
Y_{n}(\theta(\omega))=Y_{n+1}(\omega) .
$$

Denote by $\left\{\mathcal{F}_{n}\right\}_{n \geqslant 0}$ the filtration defined by

$$
\mathcal{F}_{0}=\{\emptyset, \tilde{\Omega}\} \quad \text { and } \quad \forall n \geq 1, \mathcal{F}_{n}=\sigma\left(Y_{1}, \ldots, Y_{n}\right)
$$

We consider the product space $\mathcal{G} \times \widetilde{\Omega}$ provided with its Borel $\sigma$-algebra. A map $\tilde{\theta}$ on $\mathcal{G} \times \widetilde{\Omega}$ is defined setting

$$
\forall \mathbf{e} \in \mathcal{G}, \tilde{\theta}(\mathbf{e}, \omega)=\left(\mathbf{e} Y_{1}(\omega), \theta(\omega)\right) .
$$

Theorem 3.8 says us that, for any $\mathbf{e} \in \mathcal{G}$, the $\mathcal{D}^{-}$-component of $\mathbf{e} Y_{1} \cdots Y_{n}$ converges $\widetilde{\mathbb{P}}$-almost surely towards a $\mathcal{D}^{-}$-valued random variable $\mathbf{d}^{-}(\mathbf{e},)=.\left(r_{\infty}(\mathbf{e}, \cdot)\left(\varepsilon_{0}+\varepsilon_{1}\right), N\left(x_{\infty}(\mathbf{e}, \cdot)\right)\right)$, which is $\tilde{\theta}$-invariant. If we call $\eta$ the $\operatorname{map} \omega \in \Omega \mapsto\left(\mathbf{y}_{n}(\omega)\right)_{n \in \mathbb{N}^{*}} \in \widetilde{\Omega}$, then

$$
\forall \mathbf{e} \in \mathcal{G}, \forall n \in \mathbb{N}, \mathbf{e}_{n}(\omega)=\mathbf{e y}_{1}(\omega) \cdots \mathbf{y}_{n}(\omega)=\mathbf{e} Y_{1}(\eta(\omega)) \cdots Y_{n}(\eta(\omega)), \mathbb{P}_{\mathbf{e}^{-}} \text {a.e. }
$$

Let $A \in \sigma\left(\mathbf{e}_{n}: n \geqslant 0\right)$, and consider the subset $B$ of $\mathcal{G} \times \widetilde{\Omega}$, defined by

$$
\left.1_{B}\left(\mathbf{e}_{0}, \eta(\cdot)\right)\right)=1_{A}\left(\mathbf{e}_{0}(\cdot), \ldots, \mathbf{e}_{n}(\cdot), \ldots\right) .
$$

Then,

$$
A \in \operatorname{Inv}\left(\mathbf{e}_{n}\right) \Leftrightarrow \tilde{\theta}^{-1}(B)=B .
$$

Below we prove that any Borel bounded $\tilde{\theta}$-invariant function is a Borel bounded function of $\mathbf{d}_{\infty}^{-}(\mathbf{e},$.$) . To do$ so, we shall use the following random walk version of Lemma 2.8, for which one can consult the book [19] of Neveu, Proposition V.2.4.

\section{Lemma 3.10.}

- If $Z$ is a bounded $\tilde{\theta}$-invariant random variable on $\mathcal{G} \times \widetilde{\Omega}$, then the function

$$
h(\mathbf{e})=\mathbb{E}_{\widetilde{\mathbb{P}}}[Z(\mathbf{e}, \cdot)], \mathbf{e} \in \mathcal{G}
$$


is bounded and $\mu$-harmonic. Moreover

$$
\forall n \in \mathbb{N}, \mathbb{E}_{\widetilde{\mathbb{P}}}\left[Z(\mathbf{e}, \cdot) \mid \mathcal{F}_{n}\right]=\mathbb{E}_{\widetilde{\mathbb{P}}}\left[Z\left(\mathbf{e} Y_{1} \cdots Y_{n}, \theta^{n}(\cdot)\right) \mid \mathcal{F}_{n}\right]=h\left(\mathbf{e} Y_{1} \cdots Y_{n}\right)
$$

converges $\widetilde{\mathbb{P}}$-almost surely towards $Z(\mathbf{e}, \cdot)$ (martingale convergence theorem).

- Reciprocally, if $h$ is a bounded $\mu$-harmonic function then, for any $\mathbf{e} \in \mathcal{G}$, the process $h\left(\mathbf{e} Y_{1} \cdots Y_{n}\right)$ is a bounded martingale relatively to the filtration $\left(\mathcal{F}_{n}\right)_{n \geqslant 0}$, and consequently (martingale convergence theorem), converges $\widetilde{\mathbb{P}}$-almost surely towards a random variable $Z(\mathbf{e}, \omega)$ which is $\tilde{\theta}$-invariant and satisfies

$$
h(\mathbf{e})=\mathbb{E}_{\widetilde{\mathbb{P}}}[Z(\mathbf{e}, \cdot)]
$$

Theorem 3.11 (Poisson boundary of the random walk).

- The $\sigma$-algebra generated by the random variable $\mathbf{d}^{-}(\mathbf{e}, \cdot)$ coincide with the invariant $\sigma$-algebra of $\left\{\mathbf{e}_{n}\right\}_{n \geqslant 0}$ up to $\mathbb{P}_{\mathbf{e}}$-null sets, for any $\mathbf{e} \in \mathcal{G}$.

Equivalently,

- any bounded harmonic function $h$ is of the form

$$
h(\mathbf{e})=\mathbb{E}_{\widetilde{\mathbb{P}}}\left[H\left(\mathbf{d}_{\infty}^{-}(\mathbf{e}, .)\right)\right]
$$

for some bounded Borel function $H$ on $\mathcal{N} \times \mathbb{R}$.

Lemma 3.12 (fundamental lemma). It suffices to show that any bounded $\mathcal{D}^{-}$-left invariant harmonic function is constant to prove Theorem 3.11.

Proof. Suppose that any bounded $\mathcal{D}^{-}$-left invariant harmonic function is constant, and given any bounded harmonic function $h$ let us prove that it is of the form

$$
h(\mathbf{e})=\mathbb{E}_{\widetilde{\mathbb{P}}}\left[H\left(\mathbf{d}_{\infty}^{-}(\mathbf{e}, .)\right)\right]
$$

for some bounded function $H$.

Notice that we only need to get such a representation for left uniformly continuous (LUC) functions, for if $h$ is not LUC, take $\left\{f_{n}\right\}_{n \geqslant 0}$ an approximation of unity with compact support, and set $h_{n}(\mathbf{e})=\int f_{n}(\mathbf{a}) h(\mathbf{a e}) H a a r($ da $)$. This bounded harmonic function being LUC will have a representation of the form (3.10) for some bounded function $H_{n}$ such that $\left|H_{n}\right| \leqslant\|h\|_{\infty}$. Taking a sub-sequence if necessary, we shall get

$$
h(\mathbf{e})=\lim _{n \rightarrow+\infty} h_{n}(\mathbf{e})=\lim _{n \rightarrow+\infty} \mathbb{E}\left[H_{n}\left(\mathbf{d}^{-}(\mathbf{e}, .)\right)\right]=\mathbb{E}\left[H\left(\mathbf{d}^{-}(\mathbf{e}, .)\right)\right],
$$

for some bounded function $H$. Let now suppose that $h$ is LUC.

We write $\Omega$ and $\mathbb{P}$ instead of $\tilde{\Omega}$ and $\tilde{\mathbb{P}}$. Set, for any $\mathbf{e} \in \mathcal{G}$ and $\omega \in \Omega$,

$$
Z(\mathbf{e}, \omega)=\limsup _{n} h\left(\mathbf{e} Y_{1}(\omega) \cdots Y_{n}(\omega)\right)
$$

This formula defines a Borel bounded $\tilde{\theta}$-invariant function $Z$ on $\mathcal{G} \times \Omega$, such that, for any e $\in \mathcal{G}$, the bounded martingale $h\left(\mathbf{e} Y_{1} \ldots Y_{n}\right)$ converges $\mathbb{P}$-almost surely towards $Z(\mathbf{e}, \cdot)$. We prove that there exists a bounded Borel function $H: \mathcal{D}^{-} \rightarrow \mathbb{R}$, such that one has $\mathbb{P}$-almost surely

$$
Z(\mathbf{e}, .)=H\left(\mathbf{d}^{-}(\mathbf{e}, .)\right), \forall \mathbf{e} \in \mathcal{G} .
$$

First, using the left uniform continuity of $h$, one obtains the existence of a measurable set $\Omega_{1} \subset \Omega_{1}$, of $\mathbb{P}$ probability 1 , such that the limit $Z(\mathbf{e}, \omega)$ exists for all $\omega \in \Omega_{1}$ and all $\mathbf{e} \in \mathcal{G}$. It follows that given $\mathbf{e} \in \mathcal{G}$, one defines a random variable setting

$$
\phi_{\mathbf{e}}\left(\mathbf{e}^{\prime}, \omega\right)=Z\left(\mathbf{e}\left(\mathbf{d}^{-}\left(\mathbf{e}^{\prime}, .\right)\right)^{-1} \mathbf{e}^{\prime}, \omega\right)
$$


This bounded random variable is shift invariant as a function of $\omega$ and $\mathcal{D}^{-}$-left invariant as a function of $\mathbf{e}^{\prime 14}$. So (Lem. 3.10) one defines a bounded harmonic function setting

$$
f\left(\mathbf{e}^{\prime}\right)=\mathbb{E}\left[\phi_{\mathbf{e}}\left(\mathbf{e}^{\prime}, .\right)\right]
$$

this function is $\mathcal{D}^{-}$-invariant because of the invariance of $\phi_{\mathbf{e}}\left(\mathbf{e}^{\prime},.\right)$. As such, it is constant, but depends on the parameter e; denote it by $H(\mathbf{e})$. As $f\left(\mathbf{e}_{n}\right)$ converges almost surely towards $\phi_{\mathbf{e}}\left(\mathbf{e}^{\prime}, \omega\right)^{15}$ one can suppose that one has

$$
\phi_{\mathbf{e}}\left(\mathbf{e}^{\prime}, \omega\right)=H(\mathbf{e}), \quad \forall \omega \in \Omega_{1}, \forall \mathbf{e}, \mathbf{e}^{\prime} \in \mathcal{G}
$$

that is

$$
\forall \omega \in \Omega_{1}, \forall \mathbf{e}, \mathbf{e}^{\prime} \in \mathcal{G}, \quad Z\left(\mathbf{e}\left(\mathbf{d}^{-}\left(\mathbf{e}^{\prime}, \omega\right)\right)^{-1} \mathbf{e}^{\prime}, \omega\right)=H(\mathbf{e}) .
$$

It is elementary from this formula to check that $H$ is a bounded measurable function of e $\in \mathcal{G}$. Now, taking $\mathbf{e}=\mathbf{d}_{\infty}^{-}\left(\mathbf{e}^{\prime}, \omega\right)$, formula $(3.11)$ reads

$$
\forall \omega \in \Omega_{1}, \forall \mathbf{e}^{\prime} \in \mathcal{G}, \quad Z\left(\mathbf{e}^{\prime}, \omega\right)=H\left(\mathbf{d}^{-}\left(\mathbf{e}^{\prime}, \omega\right)\right),
$$

as awaited.

It remains to show the main point.

Proposition 3.13. Any bounded $\mathcal{D}^{-}$-left invariant harmonic function is constant.

Proof. 1) The proof of this Proposition relies on a lemma which gives a sufficient condition for an element $\mathbf{x} \in \mathcal{G}$ to be a left period of any bounded harmonic function.

Lemma 3.14 (left periods of bounded harmonic functions). Suppose $\mathbf{x} \in \mathcal{G}$ is such that, for any $\mathbf{e} \in \mathcal{G}$, one can write

$$
\mathbf{x e e}_{n}=\mathbf{e e}_{n} \mathbf{x}_{n}^{\prime},
$$

where the sequence $\left\{\mathbf{x}_{n}^{\prime}\right\}_{n \geqslant 0}$ has $\mathbb{P}$-almost surely a converging (random) sub-sequence. Then, $\mathbf{x}$ is a left period for bounded harmonic functions: one has for any such $h$

$$
h(\mathbf{x e})=h(\mathbf{e}), \forall \mathbf{e} \in \mathcal{G} .
$$

Proof of Lemma 3.14. One proves this lemma in two steps.

a) We saw in Proposition 3.4 that any element of the support of $\mu$ is a "stochastic right period", meaning that

$$
\lim _{n \rightarrow+\infty} h\left(\mathbf{e}_{n} \mathbf{e}\right)=\lim _{n \rightarrow+\infty} h\left(\mathbf{e}_{n}\right),
$$

for any e in the support of $\mu$. We first show that this property holds on the bigger set

$$
T=\{\mathbf{e}=(\xi, \mathbf{g}) \in \mathcal{G} ; q(\xi) \geqslant 0\}
$$

Recall $\mathbf{e}_{n}$ is the position at time $n$ of the diffusion $\left\{\mathbf{e}_{s}\right\}_{s \geqslant 0}$ on $\mathcal{G}$ constructed in Section 2.2(c). A bounded harmonic function $h$ for the random walk being given, we know from Theorem 2.7 that it is a bounded harmonic function for any random walk of the form $\left\{\mathbf{e}_{s n}\right\}_{n \geqslant 0}, s>0$. The jump law $p_{s}(\mathbf{e}) \operatorname{Haar}(\mathrm{de})$ of this random walk having support

$$
\operatorname{supp}\left(p_{s}\right)=\left\{\mathbf{e}=(\mathbf{g}, \xi) \in \mathcal{G} ; \frac{\xi}{s} \in \operatorname{ConvHull}(\mathbb{H})\right\}
$$

\footnotetext{
${ }^{14}$ Because for any $\mathbf{d}^{-} \in \mathcal{D}^{-}$and any $\omega \in \Omega_{1}$, one has $\mathbf{d}^{-}\left(\mathbf{d}^{-} \mathbf{e}^{\prime}, \omega\right)=\mathbf{d}^{-} \mathbf{d}^{-}\left(\mathbf{e}^{\prime}, \omega\right)$.

${ }^{15}$ Since $f$ is a bounded harmonic function.
} 
one gets the $\mathbb{P}_{\mathbf{e}_{0}}$-almost sure identity

$$
\forall \mathbf{e} \in \operatorname{supp}\left(p_{s}\right), \quad \lim h\left(\mathbf{e}_{\frac{n}{p}} \mathbf{e}\right)=\lim h\left(\mathbf{e}_{\frac{n}{p}}\right)=\lim h\left(\mathbf{e}_{n}\right),
$$

applying Proposition 3.4 to the random walk $\left\{\mathbf{e}_{s n}\right\}_{n \geqslant 0}$. One can then use the right uniform continuity of $h$ to say that $\mathbb{P}_{\mathbf{e}_{0}}$-almost surely

$$
\lim h\left(\mathbf{e}_{n} \mathbf{e}\right)=\lim h\left(\mathbf{e}_{n}\right), \quad \forall \mathbf{e} \in \overline{\bigcup_{s \geqslant 0} \operatorname{supp}\left(p_{s}\right)} .
$$

The set $\overline{\bigcup_{s \geqslant 0} \operatorname{supp}\left(p_{s}\right)}$ is equal to $T$. Note that $T$ generates $\mathcal{G}$ :

$$
T T^{-1}=\mathcal{G} .
$$

b) One now proves Lemma 3.14. Let $\mathbf{x} \in \mathcal{G}$ be as in the statement and let $\left\{n_{p}\right\}_{p \geqslant 0}$ be a (random) subsequence such that $\lim \mathbf{x}_{n_{p}}^{\prime}$ exists. Using identity (3.12), write

$$
\lim \mathbf{x}_{n_{p}}^{\prime}=\mathbf{s t}^{-1}
$$

for some $\mathbf{s}, \mathbf{t} \in T$, and use the right uniform continuity of $h$ to get the $\mathbb{P}$-almost sure equalities

$$
\begin{aligned}
\lim _{n} h\left(\mathbf{x e e}_{n}\right) & =\lim _{p} h\left(\mathbf{x e e}_{n_{p}}\right)=\lim _{p} h\left(\mathbf{e}_{n_{p}} \mathbf{x}_{n_{p}}^{\prime}\right) \stackrel{\mathbf{a})}{=} \lim _{p} h\left(\mathbf{e}_{n_{p}} \mathbf{x}_{n_{p}}^{\prime} \mathbf{t}\right) \\
& =\lim _{p} h\left(\mathbf{e e}_{n_{p}} \mathbf{s}\right)=\lim _{n} h\left(\mathbf{e e}_{n} \mathbf{s}\right)=\lim _{n} h\left(\mathbf{e e}_{n}\right) .
\end{aligned}
$$

Finally take mean to obtain

$h(\mathbf{x e})=h(\mathbf{e})$.

2) The proof of Proposition 3.13 now begins. It will rely on Lemma 3.14. Let $h$ be a $\mathcal{D}^{-}$-left invariant harmonic function. To prove that $h$ is constant we shall proceed in three steps. Define the group normal sub-group $\widetilde{\mathcal{D}}=\mathbb{R}^{1, d} \times \mathcal{N}$ of $\mathcal{D}$; one has $\mathcal{D}=\widetilde{\mathcal{D}} \mathcal{A}$, and the inclusions

$$
\widetilde{\mathcal{D}} \subset \widetilde{\mathcal{D}} \mathcal{A}=\mathcal{D} \subset \mathcal{G}
$$

We prove in this point, 2 , that $h$ is $\widetilde{\mathcal{D}}$-left invariant. We shall prove in point 3 that it is $\mathcal{D}$-left-invariant, and finally that it is constant in point 4 .

i) As any $\mathbf{d} \in \widetilde{\mathcal{D}}$ can be written $\mathbf{d}=\mathbf{d}^{-}\left(\zeta\right.$, Id), for some $\mathbf{d}^{-} \in \mathcal{D}^{-}$and $\zeta \in\left\langle\varepsilon_{0}-\varepsilon_{1}, \varepsilon_{2}, \ldots, \varepsilon_{d}\right\rangle$, and since $h$ is $\mathcal{D}^{-}$-left invariant, it suffices to show that one has

$$
h(\mathbf{x e})=h(\mathbf{e}), \quad \forall \mathbf{e} \in \mathcal{G}
$$

for every $\mathbf{x} \in \mathcal{D}$ of the form $\mathbf{x}=\left(\zeta\right.$, Id), with $\zeta \in\left\langle\varepsilon_{0}-\varepsilon_{1}, \varepsilon_{2}, \ldots, \varepsilon_{d}\right\rangle$. Such $\mathbf{x}$ are in $\mathcal{D}^{+}$.

ii) For any $n \in \mathbb{N}^{*}$ we write $\mathbf{e}_{n}=\mathbf{d}_{n} \mathbf{k}_{n}=\left(\xi_{n}, N\left(x_{n}\right) A\left(t_{n}\right)\right) \mathbf{k}_{n}$. Remember that by Theorem 3.8 we know that $\left\langle\xi_{n}, \varepsilon_{0}+\varepsilon_{1}\right\rangle$ and $N\left(x_{n}\right)$ converge $\mathbb{P}$-a.s. Let $\mathbf{d}^{+}=(\zeta, I) \in \mathcal{D}^{+}$with $\zeta \in\left\langle\varepsilon_{0}-\varepsilon_{1}, \varepsilon_{2}, \ldots, \varepsilon_{d}\right\rangle$. We have

$$
\begin{aligned}
\mathbf{x d}_{n} & =\left(\zeta+\xi_{n}, N\left(x_{n}\right) A\left(t_{n}\right)\right) \\
& \left.=\mathbf{d}_{n}\left(A^{-1}\left(t_{n}\right) N^{-1}\left(x_{n}\right) \zeta\right), \mathrm{Id}\right) .
\end{aligned}
$$


Since $h$ is $\mathcal{D}^{-}$-left invariant we have to work modulo $\mathcal{D}^{-}$, and, in particular, modulo the subgroup $\left(\mathbb{R}\left(\varepsilon_{0}+\varepsilon_{1}\right), \operatorname{Id}\right)$ which is a normal subgroup of $\mathcal{D}$, because the subspace $\mathbb{R}\left(\varepsilon_{0}+\varepsilon_{1}\right)$ is invariant by the matrices of $\mathcal{N} \mathcal{A}$. So we deduce that, modulo the closed subgroup $\left(\mathbb{R}\left(\varepsilon_{0}+\varepsilon_{1}\right), \mathrm{Id}\right)$,

$$
\mathbf{x} \mathbf{d}_{n} \equiv \mathbf{d}_{n}\left(A^{-1}\left(t_{n}\right)\left(N^{-1}\left(x_{n}\right) \zeta-\left\langle N^{-1}\left(x_{n}\right) \zeta, \varepsilon_{0}+\varepsilon_{1}\right\rangle\left(\varepsilon_{0}+\varepsilon_{1}\right)\right), \mathrm{Id}\right) .
$$

Now we have

$$
\begin{aligned}
\zeta_{n} & :=A^{-1}\left(t_{n}\right)\left(N^{-1}\left(x_{n}\right) \zeta-\left\langle N^{-1}\left(x_{n}\right) \zeta, \varepsilon_{0}+\varepsilon_{1}\right\rangle\left(\varepsilon_{0}+\varepsilon_{1}\right)\right) \\
& =A^{-1}\left(t_{n}\right)\left(\left\langle N^{-1}\left(x_{n}\right) \zeta, \varepsilon_{0}-\varepsilon_{1}\right\rangle\left(\varepsilon_{0}-\varepsilon_{1}\right)+\sum_{k=2}^{d}\left\langle N^{-1}\left(x_{n}\right) \zeta, \varepsilon_{k}\right\rangle \varepsilon_{k}\right) \\
& =\mathrm{e}^{t_{n}}\left\langle N^{-1}\left(x_{n}\right) \zeta, \varepsilon_{0}-\varepsilon_{1}\right\rangle\left(\varepsilon_{0}-\varepsilon_{1}\right)+\sum_{k=2}^{d}\left\langle N^{-1}\left(x_{n}\right) \zeta, \varepsilon_{k}\right\rangle \varepsilon_{k},
\end{aligned}
$$

which shows the convergence $\mathbb{P}$-a.s. of $\zeta_{n}$. It follows from above that

$$
h\left(\mathbf{x} \mathbf{e}_{n}\right)=h\left(\mathbf{x} \mathbf{d}_{n} \mathbf{k}_{n}\right)=h\left(\mathbf{e}_{n} \mathbf{k}_{n}^{-1}\left(\zeta_{n}, \mathrm{Id}\right) \mathbf{k}_{n}\right) ;
$$

$\mathbf{k}_{n}$ moving in the compact set $\mathcal{K}$ and $\zeta_{n}$ converging, the sequence $\mathbf{k}_{n}^{-1}\left(\zeta_{n}, I\right) \mathbf{k}_{n}$ has $\mathbb{P}$-almost surely a convergent sub-sequence. By Lemma 3.14, we obtain $\forall \mathbf{e} \in \mathcal{G}, h(\mathbf{x} \mathbf{e})=h(\mathbf{e})$; this proves the $\widetilde{\mathcal{D}}$-left-invariance of $h$.

3) Recall that $\mathcal{D}=\widetilde{\mathcal{D}} \mathcal{A}$. We now show that $h$ is $\mathcal{D}$-left invariant, proving that it is $\mathcal{A}$-left invariant. Set $\mathbf{a}=(0, A(t)) \in \mathcal{A}$. Given $\mathbf{d}=(\xi, N(x)) \in \widetilde{\mathcal{D}}$, one has

$$
\mathbf{a} \mathbf{d}=(0, A(t))(\xi, N(x))=\left(A(t) \xi, N\left(\mathrm{e}^{t} x\right)\right)(0, A(t))=: \mathbf{d}^{\prime} \mathbf{a}
$$

with $\mathbf{d}^{\prime}=\left(A(t) \xi, N\left(\mathrm{e}^{t} x\right)\right) \in \mathcal{D}$. So writing $\mathbf{e}_{n}=\left(\xi_{n}, N\left(x_{n}\right)\right)\left(0, A\left(t_{n}\right)\right) \mathbf{k}_{n} \equiv \widetilde{\mathbf{d}}_{n} \mathbf{a}_{n} \mathbf{k}_{n} \in \widetilde{\mathcal{D}} \mathcal{A} \mathcal{K}$, the $\widetilde{\mathcal{D}}$-left invariance of $h(\stackrel{\bullet}{=})$ enables to write

$$
\begin{aligned}
h\left(\mathbf{x} \mathbf{e}_{n}\right) & =h\left(\mathbf{x} \widetilde{\mathbf{d}}_{n} \mathbf{a}_{n} \mathbf{k}_{n}\right)=h\left(\mathbf{d}_{n}^{\prime} \mathbf{x} \mathbf{a}_{n} \mathbf{k}_{n}\right) \stackrel{\bullet}{\doteq}\left(\mathbf{x} \mathbf{a}_{n} \mathbf{k}_{n}\right) \doteq h\left(\widetilde{\mathbf{d}}_{n} \mathbf{x} \mathbf{a}_{n} \mathbf{k}_{n}\right) \\
& =h\left(\widetilde{\mathbf{d}}_{n} \mathbf{a}_{n} \mathbf{k}_{n} \mathbf{k}_{n}^{-1} \mathbf{a}_{n}^{-1} \mathbf{x} \mathbf{a}_{n} \mathbf{k}_{n}\right)=h\left(\mathbf{e}_{n} \mathbf{k}_{n}^{-1} \mathbf{a}_{n}^{-1} \mathbf{x} \mathbf{a}_{n} \mathbf{k}_{n}\right) .
\end{aligned}
$$

As $\mathcal{A}$ is commutative, $\mathbf{a}_{n}^{-1} \mathbf{a a}_{n}=\mathbf{a}$, and

$$
h(\mathbf{a e})=h\left(\mathbf{e}_{n} \mathbf{k}_{n}^{-1} \mathbf{a k}_{n}\right) .
$$

It remains to notice that since the sequence $\left\{\mathbf{k}_{n}^{-1} \mathbf{x} \mathbf{k}_{n}\right\}_{n \geqslant 0}$ has $\mathbb{P}$-almost surely a converging (random) subsequence, Lemma 3.14 applies:

$$
h(\mathbf{a e})=h(\mathbf{e}), \quad \forall \mathbf{e} \in \mathcal{G} .
$$

4) We can now prove that $h$ is constant. Given $\mathbf{e} \in \mathcal{G}$, denote by $\pi(\mathbf{e})$ the class of $\mathbf{e}$ in $\mathcal{D} \backslash \mathcal{G}$. The application $\mathcal{K} \rightarrow \mathcal{D} \backslash \mathcal{G}, \mathbf{k} \mapsto \pi(\mathbf{k})$ is a diffeomorphism, which identifies $\mathcal{K}$ and $\mathcal{D} \backslash \mathcal{G}$. One can use it to define from the $\mathcal{D} \mathcal{A}$-left invariant function $h$ a continuous function $\underline{h}$ in $\mathcal{K}$, by the formula

$$
\underline{h}(\mathbf{k})=h(\text { dak }), \quad \forall \mathbf{d} \in \mathcal{N}, \mathbf{a} \in \mathcal{A} .
$$

Identifying $\mathcal{K}(\simeq \mathcal{D} \backslash \mathcal{G})$ and $\mathcal{N} \mathcal{A} \backslash S O_{0}(1, d)$, the space $\mathcal{K}$ appears as an $S O_{0}(1, d)$-space; for $\mathbf{k} \in \mathcal{N} \mathcal{A} \backslash S O_{0}(1, d)$ and $\mathbf{g} \in S O_{0}(1, d)$, write $\mathbf{k}$.g the right action of $\mathbf{g} \in S O_{0}(1, d)$ on $\mathbf{k} \in \mathcal{N} \mathcal{A} \backslash S O_{0}(1, d)$. 
Recall we defined $\nu()=.\mathbb{P}\left(\mathbf{g}_{1} \in.\right)$; using the ellipticity of Brownian motion $\left\{\mathbf{g}_{s}\right\}_{s} \geqslant 0$ on $S O_{0}(1, d)$ it is not difficult to show that $\nu$ charges any open set of $S O_{0}(1, d)$. Now, the harmonicity of $h$ becomes for $\underline{h}$ :

$$
\forall \mathbf{k} \in \mathcal{K}, \quad \underline{h}(\mathbf{k})=\int \underline{h}(\mathbf{k} \cdot \mathbf{g}) \nu(\mathrm{d} \mathbf{g})
$$

Let $\mathbf{k}_{0}$ be a point of $\mathcal{K}$ where $\underline{h}$ reaches its maximum. Would $\underline{h}$ not be constant we could find some $\varepsilon>0$ and some open set $\mathcal{U}$ such that one has

$$
\underline{h}(\mathbf{k})<\underline{h}\left(\mathbf{k}_{0}\right)-\varepsilon, \quad \forall \mathbf{k} \in \mathcal{U}
$$

One could then use the fact that the set $\mathcal{V}=\left\{\mathbf{g} \in S O_{0}(1, d) ; \mathbf{k}_{0} \cdot \mathbf{g} \in \mathcal{U}\right\}$, being open, has a positive $\nu$-measure to obtain a contradiction:

$$
\begin{aligned}
\underline{h}\left(\mathbf{k}_{0}\right) & =\int \underline{h}\left(\mathbf{k}_{0} \cdot \mathbf{g}\right) \nu(\mathrm{d} \mathbf{g})=\int_{\mathcal{V}} \underline{h}\left(\mathbf{k}_{0} \cdot \mathbf{g}\right) \nu(\mathrm{d} \mathbf{g})+\int_{\mathcal{V}^{c}} \underline{h}\left(\mathbf{k}_{0} \cdot \mathbf{g}\right) \nu(\mathrm{d} \mathbf{g}) \\
& \left.<\underline{h}\left(\mathbf{k}_{0}\right)-\varepsilon\right) \nu(\mathcal{V})+\underline{h}\left(\mathbf{k}_{0}\right) \nu\left(\mathcal{V}^{c}\right) \\
& <\underline{h}\left(\mathbf{k}_{0}\right) .
\end{aligned}
$$

\subsection{Invariant $\sigma$-algebra of the relativistic diffusion}

Now that we have determined the invariant $\sigma$-algebra of the random walk $\left\{\mathbf{e}_{n}\right\}_{n \geqslant 0}$, the description of the invariant $\sigma$-algebra of the relativistic diffusion is automatic: any bounded harmonic function $h$ for the relativistic diffusion extending on $\mathcal{G}$ as a harmonic function of the random walk, there exists a bounded Borel function $H$ on $\mathcal{D}^{-}$such that

$$
h\left(\left(\xi, \mathbf{g}^{0}\right)\right)=\mathbb{E}\left[H\left(\mathbf{d}_{\infty}^{-}(\mathbf{e}, .)\right)\right]
$$

where $\mathbf{e}=\left(\xi,\left(\mathbf{g}^{0}, \mathbf{g}^{1}, \ldots, \mathbf{g}^{d}\right)\right)$ is any point in $\mathcal{G}$ above $\left(\xi, \mathbf{g}^{0}\right)$. Yet it would be more satisfactory to express $\mathbf{d}_{\infty}^{-}(\mathbf{e},$.$) as a functional of the path \left\{\left(\xi_{s}, \mathbf{g}_{s}^{0}\right)\right\}_{s \geqslant 0}$ of the relativistic diffusion. This is what Theorem 3.15 makes; its proof is given in Appendix. We shall write $r_{\infty}$ and $x_{\infty}$ instead of $r_{\infty}(\mathbf{e}, \omega)$ and $x_{\infty}(\mathbf{e}, \omega)$. Recall that we denote by $\left(\rho_{s}, \sigma_{s}\right) \in \mathbb{R}_{+}^{*} \times \mathbb{S}^{d-1}$ polar coordinates of $\mathbf{g}_{s}^{0} \in \mathbb{H}$, and that $\varepsilon_{0}$ is the first vector of the canonical basis, generating the time axis.

Theorem 3.15 (asymptotic behaviour of the relativistic diffusion). Given any $\mathbf{e} \in \mathcal{G}$, one has $\mathbb{P}_{\mathbf{e}}$-almost surely

(1) $\sigma_{\infty} \in \mathbb{S}^{d-1}$ is the stereographic projection of $x_{\infty} \in \mathbb{R}^{d-1}$;

(2) $\lim _{s \rightarrow+\infty} q\left(\xi_{s}, \varepsilon_{0}+\sigma_{\infty}\right)=\frac{r_{\infty}}{1+\left|x_{\infty}\right|^{2}}$.

To prove Theorem 1.1 is now straightforward: reparameterizing $\xi_{s}$ by its time coordinate $t=\int_{0}^{s(t)} \operatorname{ch} \rho_{r} \mathrm{~d} r$, and setting $x_{t}=\int_{0}^{s(t)}\left(\operatorname{sh} \rho_{r}\right) \sigma_{r} \mathrm{~d} r$, one has

$$
\frac{\mathrm{d} x_{t}}{\mathrm{~d} t}=\left(\operatorname{th} \rho_{s(t)}\right) \sigma_{s(t)} \rightarrow \sigma_{\infty}
$$

from which the statement of Theorem 1.1 follows. 


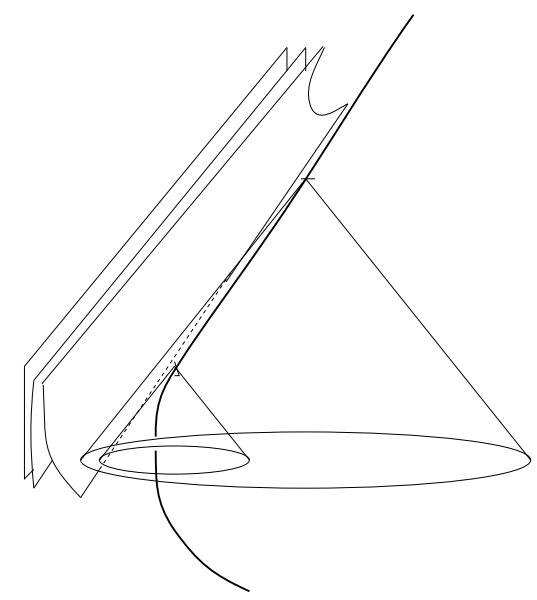

Figure 2. Past of a path.

\section{Spacetime Boundary}

If Theorem 3.11 is satisfying in that it enables to represent any bounded harmonic function on $\mathcal{G}$ in terms of the $\mathcal{G}$-space $\mathcal{D}^{-}$, its geometric content is not clear. In so far as the random walk was constructed in a canonical way from the metric defining Minkowski space, it is natural to ask if one can recover this $\mathcal{G}$-space as a geometric object accessible to someone living in $\mathbb{R}^{1, d}$. The present section brings a positive answer.

We assume throughout this section that the reader is familiar with the basic notions of Lorentzian geometry. All that is really needed is just the notion of Lorentzian manifold, of Lorentzian (infinitesimal) cone, causal, null and timelike paths, and geodesics, and conformal equivalence. All this can be found in the first pages of the book [20] of O'Neill.

\subsection{A natural boundary to spacetime}

Leaving for a moment probabilities and relativistic diffusion, this subsection is dedicated to answering this question: "How can one define a boundary to spacetime $\mathbb{R}^{1, d}$ ?" From a pedestrian point of view the information which is accessible about a point $\xi$ far away from us is its past: the set $\operatorname{Past}(\xi)=\left\{\zeta \in \mathbb{R}^{1, d} ; q(\xi-\zeta)>0\right\}$ of points from which one can emit a "signal" propagating in spacetime at a speed less than (or equal to) the speed of light up to $\xi^{16}$.

We shall call a causal path a $\mathcal{C}^{1}$ path $\gamma: I \rightarrow \mathbb{R}^{1, d}$, such that one has $q\left(\dot{\gamma}_{s}\right) \geqslant 0$ for all $s \in I$; the path will be called future oriented if its time component increases ${ }^{17}$; future oriented causal paths correspond to the preceding "signals". By past of a set we shall mean the union of the past of its elements.

Noting that points of $\mathbb{R}^{1, d}$ are characterized by their past ${ }^{18}$ it seems natural to say that two future-oriented causal curves $\gamma$ and $\gamma^{\prime}$, leaving every compact, converge towards the same point at infinity if $\gamma_{s}$ and $\gamma_{s}^{\prime}$ eventually have the "same" past:

$$
\gamma \sim \gamma^{\prime} \quad \text { if } \bigcup_{s \geqslant 0} \operatorname{Past}\left(\gamma_{s}\right)=\bigcup_{s \geqslant 0} \operatorname{Past}\left(\gamma_{s}^{\prime}\right) .
$$

This equivalence relation defines the causal boundary of $\mathbb{R}^{1, d}$.

\footnotetext{
${ }^{16}$ The notation usually used for $\operatorname{Past}(\xi)$ is $J^{-}(\xi)$.

${ }^{17}$ Remember the set $\{q>0\}$ has two components.

${ }^{18} \xi=\xi^{\prime}$ iff $\operatorname{Past}(\xi)=\operatorname{Past}\left(\xi^{\prime}\right)$.
} 
If geometric intuition leads naturally to this notion of boundary, it is not that easy to formulate correctly ${ }^{19}$, and certainly not practical at first sight. We are going to bypass this difficulty using the fact that past cones used to define that boundary are conformal objects. This will allow us to embed $\left(\mathbb{R}^{1, d}, q\right)$ conformally in a compact Lorentzian manifold $\left(\mathbf{E i n}_{2+d}, \widehat{q}\right)$ that we are going to introduce in Section 4.1.1. This space $\mathbf{E i n}_{2+d}$ has the property that the topological boundary $\overline{\mathbb{R}^{1, d}} \backslash \mathbb{R}^{1, d}$ of $\mathbb{R}^{1, d}$ in $\mathbf{E i n}_{2+d}$ can be identified with its causal boundary, as defined above. The space $\left(\mathbf{E i n}_{2+d}, \widehat{q}\right)$ is called Einstein universe. This spacetime is a classical and fundamental object in relativity, for it plays in Lorentzian geometry the role that spheres plays in Riemannian geometry: any simply connected constant curvature Lorentzian manifold appears as an open set of this space. We describe the classical construction of $\mathbf{E i n}_{2+d}$ and some of its elementary properties in the next section ${ }^{20}$; the conformal embedding of $\mathbb{R}^{1, d}$ in $\mathbf{E i n}_{2+d}$ will be explicitly written in Section 4.1.2.

\subsubsection{Einstein universe}

a) Construction

Definition 4.1. Endow $\mathbb{R}^{2+(1+d)}$ with the quadratic form

$$
q^{2,1+d}(x)=-2 x^{0} x^{2+d}+\left(x^{1}\right)^{2}-\left(x^{2}\right)^{2}-\cdots-\left(x^{1+d}\right)^{2},
$$

expressed in the canonical basis $\left(\widetilde{\varepsilon}_{0}, \widetilde{\varepsilon}_{1}, \ldots, \widetilde{\varepsilon}_{1+d}, \widetilde{\varepsilon}_{2+d}\right)$ of $\mathbb{R}^{2+(1+d)}$. Endowed with this quadratic form, $\mathbb{R}^{2+(1+d)}$ will be denoted $\mathbb{R}^{2,1+d}$. Denote by $C^{2,1+d}$ the lightcone of $\mathbb{R}^{2,1+d}$, made up of all vectors with $q^{2,1+d}$-null norm. $C^{2,1+d} \backslash\{0\}$ is a smooth manifold. Its tangent space at a point $v \in C^{2,1+d} \backslash\{0\}$ is denoted by $T_{v} C^{2,1+d}$.

Let $\pi: C^{2,1+d} \rightarrow \mathbb{R} \mathbb{P}^{2+d}$ be the restriction to $C^{2,1+d}$ of the canonical projection of $\mathbb{R}^{2+(1+d)} \backslash\{0\}$ in $\mathbb{R} \mathbb{P}^{2+d}$. Write

$$
\operatorname{Ein}_{1+d} \equiv \pi\left(C^{2,1+d}\right)
$$

this is a hypersurface of $\mathbb{R P}^{2+d}$. Let $v \in C^{2,1+d}$ and set $p=\pi(v)$. The restriction of the quadratic form $q^{2,1+d}$ to $T_{v} C^{2,1+d}$ has signature $(0,+,-, \cdots,-)$, where 0 is for the $v$-direction. Since $v$ is in the kernel of $\pi, T_{p} \operatorname{Ein}_{1+d}$ bears a Lorentzian cone, $\widetilde{C}(p)$, image of the lightcone $\underline{C}(v)$ of $q_{\mid T_{v} C^{2,1+d}}^{2,1+d}$, by $T_{v} \pi$. This cone $\widetilde{C}(p)$ is well defined:

$$
T_{v^{\prime}} \pi\left(\underline{C}\left(v^{\prime}\right)\right)=T_{v} \pi(\underline{C}(v)) \text {, if } \pi\left(v^{\prime}\right)=\pi(v) .
$$

It depends smoothly on $p$. This smooth distribution of Lorentzian cones gives $\mathbf{E i n}_{1+d}$ a natural conformal Lorentzian structure.

Definition 4.2. Einstein universe is this Lorentzian manifold together with its conformal structure:

$$
\left(\operatorname{Ein}_{1+d},\{\widetilde{C}(p)\}_{p \in \operatorname{Ein}_{1+d}}\right) .
$$

This construction of $\mathbf{E i n}_{1+d}$ makes it clear that its group of conformal transforms contains the projective group $P O(2,1+d)$ of $q^{2,1+d}$-orthogonal transforms. The situation is clarified by the following Liouville type theorem ${ }^{21}$.

Theorem 4.3 (Liouville). - $P O(2,1+d)$ acts transitively on $\mathbf{E i n}_{1+d}$ and respects its conformal structure.

- $P O(2,1+d)$ acts transitively on the bundle of lightlike directions over $\mathbf{E i n}_{1+d}$.

- Any local conformal transformation of $\mathbf{E i n}_{1+d}$ is the restriction of the action on $\mathbf{E i n}_{1+d}$ of a unique element of $P O(2,1+d)$.

a) Lightlike geodesics and light cone of a point $\bullet$ In the framework of conformal Lorentzian geometry, the notion of geodesic is not well defined generally; yet, that of lightlike geodesic is a well defined geometric notion.

\footnotetext{
${ }^{19}$ See [12].

${ }^{20}$ Consult for instance the article [15], or the book [6] of Beem and Ehrlich.

${ }^{21}$ See [11] for a proof.
} 


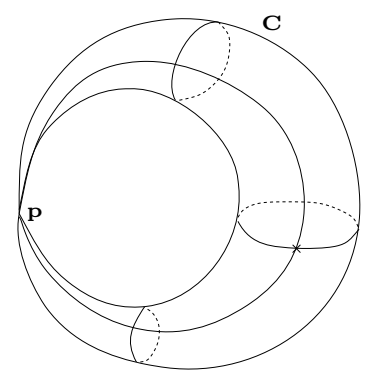

Figure 3. The lightcone $\mathbf{C}(p)$ of $p$.

Proposition 4.4. Let $g_{0}$ be a Lorentzian metric on $\mathbf{E i n}_{1+d}$ with the $\widetilde{C}(p)$ 's as null cones. Let $f: \operatorname{Ein}_{1+d} \rightarrow \mathbb{R}$ be a smooth map, and $\mathrm{e}^{2 f} g_{0}$ a conformal metric on $\mathbf{E i n}_{1+d}$. Any lightlike $g_{0}$-geodesic $\gamma: I \rightarrow \mathbf{E i n}_{1+d}$ can be reparameterised to give a $\left(\mathrm{e}^{2 f} g_{0}\right)$ - lightlike geodesic.

Proof. Let $\nabla$ and $\nabla^{\prime}$ be the Levi-Civita connection associated with $g_{0}$ and $\mathrm{e}^{2 f} g_{0}$ respectively. The statement is a direct consequence of the following identity

$$
\nabla_{V}^{\prime} W=\nabla_{V} W+(V . f) W+(W . f) V-g_{0}(V, W) \nabla f,
$$

holding for every vector fields $V, W$ on $\operatorname{Ein}_{1+d}$, and where $\nabla f$ is the gradient of $f$ with respect to the metric $g_{0}$.

This proposition justifies that we should introduce the

Definition 4.5. A 1-dimensional manifold $\Gamma \subset \mathbf{E i n}_{1+d}$ is said to be a lightlike geodesic if any point $\xi$ of $\Gamma$ has a neighbourhood $\mathcal{U}$ such that $\Gamma \cap \mathcal{U}$ is of the form $\gamma(I)$ for some $\left(\mathrm{e}^{2 f} g_{0}\right)$-geodesic $\gamma: I \rightarrow \mathbf{E i n}_{1+d}$.

Fortunately, the high homogeneity of the space enables to give an elementary description of lightlike geodesics, proved in Appendix.

Proposition 4.6. Any lightlike geodesic running through the point $\pi(v)$ is of the form $\pi\left(\left\langle v, v^{\prime}\right\rangle \cap C^{2,1+d}\right)$, where $v^{\prime} \in T_{v} C^{2,1+d}$ is a null vector. In other words, the non parameterized lightlike geodesics are the projections in $\operatorname{Ein}_{1+d}$ of totally degenerated planes of $\mathbb{R}^{2,1+d}$; they are topological circles.

Definition 4.7. Given some point $p$ in $\mathbf{E i n}_{1+d}$, the set of all lightlike geodesics running through $p$ is called the lightcone of $p$ and denoted $\mathbf{C}(p)$.

One deduces from the preceding proposition that if $p=\pi(v)$ and $v^{\perp}$ is the orthogonal of $v$ with respect to $q^{2,1+d}$, then

$$
\mathbf{C}(p)=\pi\left(C^{2,1+d} \cap v^{\perp}\right) .
$$

$\widetilde{C}(p)$ is the infinitesimal version of $\mathbf{C}(p)$. For example, if $p=\pi\left(\widetilde{\varepsilon}_{0}\right)$,

$$
\mathbf{C}(p)=\pi\left(C^{2,1+d} \cap\left\{x_{2+d}=0\right\}\right) .
$$

One sees on that formula that $\mathbf{C}(p)$ is a compact subset of Einstein universe ${ }^{22}$ with a singularity at $p$, such that $\mathbf{C}(p) \backslash\{p\}$ is a hypersurface, diffeomorphic to $\mathbb{R} \times \mathbb{S}^{d-1}$. Note that $\mathbf{E i n}_{1+d} \backslash \mathbf{C}(p)$ is dense in $\mathbf{E i n}_{1+d}$ and that $\mathbf{C}(p) \backslash\{p\}$ is foliated by lightlike geodesics: any point of $\mathbf{C}(p) \backslash\{p\}$ belongs to a unique lightlike geodesic.

\footnotetext{
${ }^{22}$ Einstein universe being a closed subset of the compact manifold $\mathbb{R} \mathbb{P}^{2+d}$ is compact. $\mathbf{C}(p)$ is a closed subset of Einstein universe.
} 


\subsubsection{Where does Minkowski space hide?}

Set $\mathbf{p}=\pi\left(\widetilde{\varepsilon}_{0}\right) \in \mathbf{E i n}_{1+d}$. Recall $\left(\varepsilon_{0}, \ldots, \varepsilon_{d}\right)$ denotes the canonical basis of $\mathbb{R}^{1, d}$. The following proposition provides an explicit conformal embedding of $\mathbb{R}^{1, d}$ in $\mathbf{E i n}_{1+d}$. It is taken from [11].

Proposition 4.8. $\mathbb{R}^{1, d}$ is conformally equivalent to the dense open set $\mathbf{E i n}_{1+d} \backslash \mathbf{C}(\mathbf{p})$. In that sense, Ein $1+d$ provides a compactification of $\mathbb{R}^{1, d}$ in which $\mathbf{C}(\mathbf{p})$ is its boundary.

Proof. Let $i:\left(\mathbb{R}^{1, d}, q\right) \rightarrow\left(\mathbb{R}^{2,1+d}, q^{2,1+d}\right)$ be the linear identification of $\mathbb{R}^{1, d}$ to $\left\langle\widetilde{\varepsilon}_{1}, \ldots, \widetilde{\varepsilon}_{1+d}\right\rangle$ sending $\varepsilon_{i}$ on $\widetilde{\varepsilon}_{i+1}$; this is an isometry. The map

$$
j: x \in \mathbb{R}^{1, d} \mapsto q(x, x) \widetilde{\varepsilon}_{0}+2 i(x)+\widetilde{\varepsilon}_{2+d} \in C^{2,1+d}
$$

is well defined and maps the lightcone of $\mathbb{R}^{1, d}$ to the lightcone of $T_{j(x)} C^{2,1+d}$. The map $\pi$ being injective on $j\left(\mathbb{R}^{1, d}\right)$, the application $\pi \circ j$ is a conformal equivalence between $\mathbb{R}^{1, d}$ and $\pi \circ j\left(\mathbb{R}^{1, d}\right) \subset \mathbf{E i n}_{1+d}$; it is called a stereographic projection. To prove that $\pi \circ j\left(\mathbb{R}^{1, d}\right)$ is dense in $\operatorname{Ein}_{1+d}$, note that the only points of $\mathbf{E i n}_{1+d}$ that are not in the image of $\pi \circ j$ are the projection in $\mathbf{E i n}_{1+d}$ of the points $x$ of $C^{2,1+d}$ with $x_{2+d}=0$, i.e. $\mathbf{C}(\mathbf{p})$, as noticed in (4.1).

From now on, we identify $\mathbb{R}^{1, d}$ and its stereographic projection $\pi \circ j\left(\mathbb{R}^{1, d}\right) \subset \mathbf{E i n}_{1+d}$. We shall write $\mathbf{C}$ for $\mathbf{C}(\mathbf{p})$.

\subsubsection{Spacetime boundary and bounded harmonic functions}

The stereographic projection is the adequate tool to identify the causal boundary of $\mathbb{R}^{1, d}$ as the set $\mathbf{C}$. The following theorem gives a much more precise description saying among other things that $\mathbf{C} \backslash\{\mathbf{p}\}$ can be identified from the inside of $\mathbb{R}^{1, d}$ and characterizing convergence towards some point of $\mathbf{C} \backslash\{\mathbf{p}\}$. Although this theorem is essentially well known to specialists, no proof being available in the literature, we prove it in Appendix.

\section{Theorem 4.9.}

1. For any non lightlike geodesic $\xi+\mathbb{R} v$ of $\mathbb{R}^{1, d}, \xi+$ tv converges to $\mathbf{p}$ as $t \rightarrow+\infty$.

2. Any point in $\mathbf{C} \backslash\{\mathbf{p}\}$ is the limit of a lightlike geodesic of $\mathbb{R}^{1, n-1}$. More precisely,

- Let $v \in \mathbb{R}^{1, d}$ be an isotrope vector. There is a lightlike geodesic $\Delta_{v}$ of $\mathbf{C}$ such that for every lightlike geodesic $\xi+\mathbb{R} v$, with direction $v, \xi+$ tv converges towards a point of $\Delta_{v} \backslash\{\mathbf{p}\}$ as $t \rightarrow+\infty$. This lightlike geodesic of $\mathbf{E i n}_{1+d}$ depends only on the direction $\mathbb{R} v$ of $v$.

Identify the set of null directions with $\mathbb{S}^{d-1}$ : say that the vector $v$ has direction $\sigma$ if $\mathbb{R} v=\mathbb{R}\left(\varepsilon_{0}+\sigma\right)$, $\sigma \in \mathbb{S}^{d-1}$. Write $\Delta_{\sigma}$ for $\Delta_{v}$ if $v$ has direction $\sigma$.

Now, given $\sigma \in \mathbb{S}^{d-1}$ and a null vector $v=\varepsilon_{0}+\sigma \in \mathbb{R}^{1, d}$ with direction $\sigma$,

- two v-directed geodesics $\xi+\mathbb{R} v$ and $\xi^{\prime}+\mathbb{R} v$ converge to the same point iff $\xi^{\prime}$ is in the affine hyperplane $\xi+v^{\perp}$.

The function $q(v,$.$) is constant on \xi+v^{\perp}$, note $\ell \in \mathbb{R}$ this constant. We note $p_{\sigma}(\ell) \in \Delta_{\sigma}$ the limit of $\xi+\mathbb{R} v$.

- Any point of $\Delta_{\sigma}$ is the limit of a lightlike geodesic $\xi+\mathbb{R}\left(\varepsilon_{0}+\sigma\right)$, for some $\xi \in \mathbb{R}^{1, d}$.

3. A natural parameterization of $\mathbf{C} \backslash\{\mathbf{p}\}-$ The map

$$
(\sigma, \ell) \in \mathbb{S}^{d-1} \times \mathbb{R} \mapsto p_{\sigma}(\ell) \in \mathbf{C} \backslash\{\mathbf{p}\}
$$

is a diffeomorphism.

4. (a) Every timelike path $\left\{\gamma_{s}\right\}_{s \in I}$ in $\mathbb{R}^{1, d}$, future-oriented and inextensible ${ }^{23}$, converges toward a point of $\mathbf{C}$.

(b) Let $\sigma \in \mathbb{S}^{d-1}$ and $\ell \in \mathbb{R}$.

$$
\gamma_{s} \underset{s \rightarrow+\infty}{\longrightarrow} p_{\sigma}(\ell) \text { iff } q\left(\gamma_{s}, \varepsilon_{0}+\sigma\right) \underset{s \rightarrow+\infty}{\longrightarrow} \ell
$$

\footnotetext{
${ }^{23}$ That is, $I$ is an interval of $\mathbb{R}$, the time component $\gamma_{t}^{0}$ of $\gamma_{t}$ increases, and $\gamma: I \rightarrow \mathbb{R}^{1, d}$ has no proper $\mathcal{C}^{1}$ extension.
} 


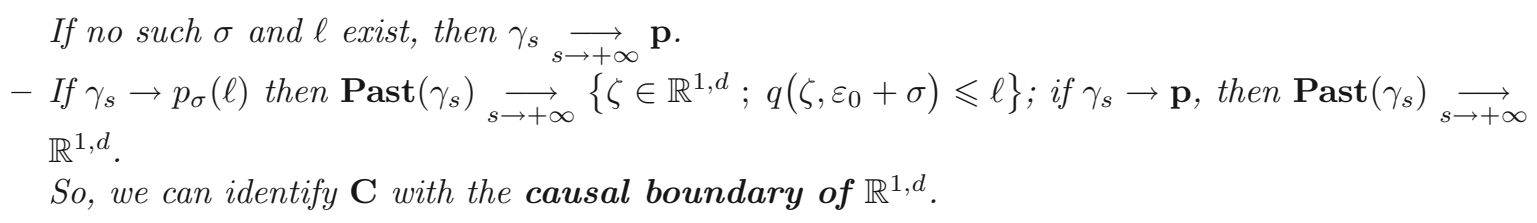

Comparing assertion 4(b) and Theorem 1.1 brings an answer to geometer's question formulated in the introduction.

\section{Theorem 4.10.}

- The $\mathbb{R}^{1, d}$-part $\left\{\xi_{s}\right\}_{s \geqslant 0}$ of the relativistic diffusion $\mathbb{P}_{(\xi, \dot{\xi})}$-converges almost surely towards some random point $\xi_{\infty}$ of $\mathbf{C} \backslash\{\mathbf{p}\}$, for any initial starting point $(\xi, \dot{\xi})$.

- The $\sigma$-algebra generated by $\xi_{\infty}$ coincide with the tail $\sigma$-algebra of $\left\{\xi_{s}\right\}_{s \geqslant 0}$ up to $\mathbb{P}_{(\xi, \dot{\xi})}$-null sets.

The boundary $\mathbf{C}$ describing the space of lightlike geodesics, we shall resume this theorem as the relativistic diffusion eventually behaves as a lightlike geodesic, a good conclusion where to stop.

\section{Appendix}

This appendix contains the different proofs that were not written in the core of the article to make it more readable. We prove Proposition 2.6 concerning the properties of the jump law of the random walk, Theorem 3.15 reformulating the algebraic result on the invariant $\sigma$-algebra of the relativistic diffusion in terms of spacetime quantities, and Theorem 4.9 identifying the causal boundary of $\mathbb{R}^{1, d}$.

\subsection{Proof of Proposition 2.6 on the properties of the law of $\left\{\mathrm{e}_{s}\right\}_{s \geqslant 0}$}

a) - 1. This point comes from the fact that the infinitesimal generator $\widetilde{L}$ of $\left\{\mathbf{e}_{s}\right\}_{s} \geqslant 0$ is a hypoelliptic differential operator: see [4], Proposition 10.

2. We determine the support of the probability $p_{s}$. Fix $s>0$ and denote by $\delta$ any left-invariant distance on $S O_{0}(1, d)$.

1) As the Brownian motion $\left\{\mathbf{g}_{s}\right\}_{s} \geqslant 0$ on $S O_{0}(1, d)$ is an elliptic diffusion, for any continuous (deterministic) path $\left\{\gamma_{r}\right\}_{0 \leqslant r \leqslant s}$ in $S O_{0}(1, d)$ and any $\varepsilon>0$ we have

$$
\mathbb{P}_{\mathbf{g}_{0}}\left(\sup _{0 \leqslant r \leqslant s} \delta\left(\mathbf{g}_{r}, \gamma_{r}\right) \leqslant \varepsilon\right)>0
$$

So, the support of $P_{s}\left(\left(\mathbf{g}_{0}, \xi_{0}\right),.\right)$ is the closure of the set of points $(\underline{\mathbf{g}}, \zeta) \in \mathcal{G}$ of the form $\underline{\mathbf{g}}=\gamma_{s}$ and $\zeta=$ $\zeta_{0}+\int_{0}^{s} \gamma_{r} \mathrm{~d} r$ for some continuous path $\left\{\underline{\mathbf{g}}_{r}\right\}_{r \leqslant s}$ in $S O_{0}(1, d)$.

2) Besides, as $\left\{\mathbf{g}_{s}\right\}_{s} \geqslant 0$ can go from one open set of $S O_{0}(1, d)$ to any other one in an arbitrarily small amount of time with positive probability, we see that the heart of the proof is to show that $\left\{\frac{1}{s} \int_{0}^{s} \gamma_{r}^{0} \mathrm{~d} r ; \gamma:[0, s] \rightarrow\right.$ $S O_{0}(1, d)$, continuous $\}=\operatorname{ConvHull}(\mathbb{H})$.

3) Taking constant $\gamma$ 's gives

$$
\operatorname{ConvHull}(\mathbb{H}) \subset\left\{\frac{1}{s} \int_{0}^{s} \gamma_{r}^{0} \mathrm{~d} r ; \gamma:[0, s] \stackrel{\mathcal{C}^{0}}{\rightarrow} \mathbb{H}\right\} .
$$

4) Conversely, the convexity of ConvHull( $\mathbb{H})$ implies that for any $n \geqslant 1$,

$$
\frac{1}{n} \sum_{j=0}^{n-1} \gamma_{\frac{j s}{n}}^{0} \in \operatorname{ConvHull}(\mathbb{H})
$$


since each $\gamma_{\frac{j s}{n}}^{0}$ belongs to $\mathbb{H} \subset \operatorname{ConvHull}(\mathbb{H})$. As $\frac{1}{n} \sum_{j=0}^{n-1} \gamma_{\frac{j s}{n}}^{0} \underset{n \rightarrow+\infty}{\longrightarrow} \frac{1}{s} \int_{0}^{s} \gamma_{r}^{0} \mathrm{~d} r$, the result follows.

3. Actually we can prove a little more.

Proposition 5.1. The probability $p_{s}(\mathbf{a}) \operatorname{Haar}(\mathrm{d} \mathbf{a})$ has moments of any order.

Proof. We can take $s=1$ without loss of generality, and write $\mu$ instead of $p_{1}(\mathbf{a}) \operatorname{Haar}(\mathrm{d} \mathbf{a})$. Let $p \geqslant 1$. Given any neighbourhood $V$ of $\operatorname{Id}_{\mathcal{G}}$, we need to show that the associated gauge $f_{V}$ is in $\mathbb{L}^{p}(\mu)$. As they are all equivalent (Prop. 3.2) we can choose a particular $V$. To describe it, identify $S O_{0}(1, d)$ with the set of orthonormal frames on $\mathbb{H}$. Set

$$
\widetilde{V}=\left\{\left(\mathbf{g}^{0}, \mathcal{R}\right) \in \mathbb{O H} ; d\left(\mathbf{g}^{0}, \varepsilon_{0}\right)<1\right\}^{24} ;
$$

this open set of $S O_{0}(1, d)$ satisfies the identity

$$
\widetilde{V}^{n}=\left\{\left(\mathbf{g}^{0}, \mathcal{R}\right) \in \mathbb{O H} ; \mathrm{d}\left(\mathbf{g}^{0}, \varepsilon_{0}\right)<n\right\} .
$$

Define $V$ as the product

$$
V=\widetilde{V} \times B
$$

where $B$ is the unit Euclidean ball of $\mathbb{R}^{1+d}$.

We shall prove that one has

$$
\sum_{n \geqslant 2} \mu\left(\left(V^{n}\right)^{c}\right) n^{p}<+\infty
$$

which implies that

$$
\int f_{V}(\mathbf{e})^{p} \mu(\mathrm{d} \mathbf{e})=\mu(V)+\sum_{n \geqslant 2} \mu\left(V^{n} \backslash V^{n-1}\right) n^{p}<\infty .
$$

The proof relies on the following elementary fact, whose proof is left to the reader.

Lemma 5.2. Let $q \geqslant 1, \underline{g} \in \widetilde{V}^{q}$. The set $\left\{\underline{\mathbf{g g}} ; \mathbf{g} \in \widetilde{V}^{2 q}\right\}$ contains $\widetilde{V}^{q}$.

Notations. We denote by $\left[\frac{n}{9}\right]$ the integer part of $\frac{n}{9}$, and set $q_{n}=\left[\frac{n}{9}\right]-1$.

Let $n \geqslant 18$. As the point

$$
\left(\mathbf{g}_{1}, 0\right) \cdots\left(\mathbf{g}_{q_{n}}, 0\right)\left(\mathbf{g}_{q_{n}+1}, \xi\right)\left(\mathbf{g}_{q_{n}+2}, 0\right) \cdots\left(\mathbf{g}_{3 q_{n}}, 0\right)\left(\mathbf{g}_{3 q_{n}+1}, \xi^{\prime}\right)\left(\mathbf{g}_{3 q_{n}+2}, 0\right) \cdots\left(\mathbf{g}_{9 q_{n}+3}, 0\right),
$$

belongs to $\widetilde{V}^{n}$ for any $\xi, \xi^{\prime} \in B$, and is equal to

$$
\left(\mathbf{g}_{1} \cdots \mathbf{g}_{9 q_{n}+3}, \mathbf{g}_{1} \cdots \mathbf{g}_{q_{n}} \xi+\mathbf{g}_{1} \cdots \mathbf{g}_{q_{n}} \mathbf{g}_{q_{n}+1} \cdots \mathbf{g}_{3 q_{n}} \xi^{\prime}\right)
$$

we conclude from Lemma 5.2 that

$$
\widetilde{V}^{3 q_{n}+1}\left(\widetilde{V}^{q_{n}} \cdot B+\widetilde{V}^{q_{n}} \cdot B\right) \subset V^{n 25}
$$

The inclusion

$$
\widetilde{V}^{q_{n}}\left(\widetilde{V}^{q_{n}} \cdot B+\widetilde{V}^{q_{n}} \cdot B\right) \subset V^{n}
$$

will be sufficient to meet our purpose.

\footnotetext{
${ }^{24}$ The notation $\left(\mathbf{g}^{0}, \mathcal{R}\right) \in \mathbb{O} \mathbb{H}$ means that $\mathbf{g}^{0} \in \mathbb{H}$ and $\mathcal{R}$ is an orthonormal basis of $T_{\mathbf{g}^{0}} \mathbb{H} ; d$ is the hyperbolic distance in $\mathbb{H}$.

${ }^{25}$ We write $\widetilde{V}^{q_{n}} \cdot B$ for $\left\{\mathbf{g}(b) \in \mathbb{R}^{1, d} ; \mathbf{g} \in \widetilde{V}^{q_{n}}, b \in B\right\}$, and $A+B=\{a+b ; a \in A, b \in B\}$.
} 
So,

$$
\mu\left(\left(V^{n}\right)^{c}\right) \leqslant \mu\left(\left(\widetilde{V}^{q_{n}}\left(\widetilde{V}^{q_{n}} \cdot B+\widetilde{V}^{q_{n}} \cdot B\right)\right)^{c}\right) \leqslant \mu\left(\left(\widetilde{V}^{q_{n}}\right)^{c} \mathbb{R}^{1, d}\right)+\mu\left(\left\{(\mathbf{g}, \xi) \in \mathcal{G} ; \xi \notin\left(\widetilde{V}^{q_{n}} \cdot B+\widetilde{V}^{q_{n}} \cdot B\right)\right\}\right) .
$$

We majorize both terms of the right hand side of inequality (5.2) separately.

a) The first one is equal to $\mathbb{P}\left(\mathbf{g}_{1} \notin \widetilde{V}^{q_{n}}\right)$. Noting $\left(\rho_{1}, \theta_{1}\right)$ the polar coordinates of $\mathbf{g}_{1}^{0}$,

$$
\widetilde{\mathbb{P}}\left(\mathbf{g}_{1} \notin \widetilde{V}^{q_{n}}\right) \leqslant \mathbb{P}\left(\rho_{1} \geqslant q_{n}\right) \leqslant \mathbb{P}\left(\sup _{s \in[0,1]} \rho_{s} \geqslant q_{n}\right) .
$$

We estimate this probability using the comparison theorem on the equation

$$
\rho_{s}=w_{s}+\int_{0}^{s} \frac{d-1}{2} \operatorname{coth}\left(\rho_{r}\right) \mathrm{d} r
$$

noting that $\operatorname{coth}(\rho) \leqslant 2$ as soon as $\rho \geqslant 2$. Precisely, if one notes $w^{(d-1), 2}$ a Brownian motion with $\operatorname{drift}(d-1)$, started from 2 , one has

$$
\sup _{s \in[0,1]} \rho_{s} \leqslant \sup _{s \in[0,1]} w_{s}^{(d-1), 2} .
$$

Denote by $\mathbb{P}_{2}^{(d-1)}$ the law of $w^{(d-1), 2}$. One can use formula 1.1.4, in [7], p. 197, to conclude from (5.3) that

$$
\mathbb{P}\left(\sup _{s \in[0,1]} \rho_{s} \geqslant q_{n}\right) \leqslant \mathbb{P}_{2}^{(d-1)}\left(\sup _{s \in[0,1]} w_{s}^{(d-1), 2} \geqslant q_{n}\right) \leqslant \frac{1}{2} f\left(\frac{q_{n}-d-1}{\sqrt{2}}\right)+\frac{1}{2} \mathrm{e}^{(d-1)\left(q_{n}-2\right)} f\left(\frac{q_{n}-3+d}{\sqrt{2}}\right)
$$

with $f(x)=\frac{\mathrm{e}^{-x^{2}}}{\sqrt{\pi} x}$. The convergence

$$
\sum_{n \geqslant 2} \mathbb{P}\left(g_{1} \notin \widetilde{V}^{q_{n}}\right) n^{p}<\infty
$$

follows.

b) The second term of the right member of inequality (5.2) is equal to $\mathbb{P}\left(\xi_{1} \notin \widetilde{V}^{q_{n}} \cdot B+\widetilde{V}^{q_{n}} \cdot B\right)$.

To deal with it, we use the following two lemmas; the proof of the first one is elementary.

Write $B_{\mathbb{H}}\left(\varepsilon_{0}, R\right)=\left\{\mathbf{g}^{0} \in \mathbb{H} ; d\left(\varepsilon_{0}, \mathbf{g}^{0}\right) \leqslant R\right\}$, where $d$ denotes the hyperbolic distance in $\mathbb{H}$.

Lemma 5.3. Let $R>0$. For any continuous path $\left\{\mathbf{g}_{s}^{0}\right\}_{s \in[0,1]}$ contained in $B_{\mathbb{H}}\left(\varepsilon_{0}, R\right)$, one has

$$
\int_{0}^{1} \mathbf{g}_{s}^{0} \mathrm{~d} s \in \operatorname{ConvHull}\left(B_{\mathbb{H}}\left(\varepsilon_{0}, R\right)\right) \subset \mathbb{R}^{1, d} .
$$

Lemma 5.4. ConvHull $\left(B_{\mathbb{H}}\left(\varepsilon_{0}, q_{n}\right)\right) \subset \widetilde{V}^{q_{n}} \cdot B+\widetilde{V}^{q_{n}} \cdot B$

Proof of Lemma 5.4. In dimension $d=1$, we see on Figure 4 that the inclusion of the statement holds: a point of $\mathbb{H}$ in $B_{\mathbb{H}}\left(\varepsilon_{0}, q_{n}\right)$ being of the form $g\left(\varepsilon_{0}\right)$ for some $g \in \widetilde{V}^{q_{n}}$, any point in the convex hull in $\mathbb{R}^{1, d}$ of $B_{\mathbb{H}}\left(\varepsilon_{0}, q_{n}\right)$ can be written as $g\left(\varepsilon_{0}\right)+g^{\prime}\left(s \varepsilon_{0}\right)$, where $g^{\prime}$ is the hyperbolic rotation of angle $-q_{n}$ and $s \in[0,1]$.

In bigger dimension, any point $\xi$ of $\operatorname{ConvHull}\left(B_{\mathbb{H}}\left(\varepsilon_{0}, q_{n}\right)\right)$ is in the plane $\left\langle\varepsilon_{0}, \xi\right\rangle$, where the 2-dimensional result applies. 


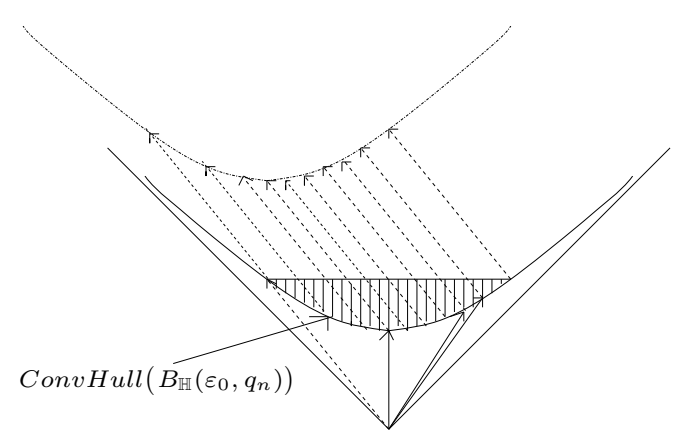

Figure 4 . The dashed zone is in $\widetilde{V}^{q_{n}} \cdot B+\widetilde{V}^{q_{n}} \cdot B$.

One deduces from Lemmas 5.3 and 5.4 that

$$
\mu\left(\left\{(\mathrm{g}, \xi) \in \mathcal{G} ; \xi \notin \widetilde{V}^{q_{n}} \cdot B+\widetilde{V}^{q_{n}} \cdot B\right) \leqslant \mathbb{P}\left(\sup _{s \in[0,1]} \rho_{s} \geqslant q_{n}\right) .\right.
$$

It remains to use formula 1.1.4 of [7] to obtain

$$
\sum_{n \geqslant 2} \mathbb{P}\left(\xi_{1} \notin \widetilde{V}^{q_{n}} \cdot B+\widetilde{V}^{q_{n}} \cdot B\right) n^{p}<\infty .
$$

Together with (5.4) and (5.2), this inequality proves (5.1).

\subsection{Proof of Theorem 3.15 on the asymptotic behaviour of the relativistic diffusion}

1. We first show that the asymptotic direction $\sigma_{s}$ of the speed $\mathbf{g}_{s}^{0}$ of the relativistic diffusion is the stereographic projection of the vector $x_{\infty} \in \mathbb{R}^{d-1}$ appearing in $\mathbf{d}_{\infty}^{-}$.

On the one hand, the expression

$$
\mathbf{g}_{s}^{0}=\mathbf{g}_{s} \varepsilon_{0}=N\left(x_{s}\right) A\left(t_{s}\right) K_{s} \varepsilon_{0}=N\left(x_{s}\right) A\left(t_{s}\right) \varepsilon_{0}=N\left(x_{s}\right)\left(\begin{array}{c}
\operatorname{ch} t_{s} \\
\operatorname{sh} t_{s} \\
(0)
\end{array}\right)
$$

gives the coordinates of $\mathbf{g}_{s}^{0}$ in the canonical basis:

$$
\mathbf{g}_{s}^{0}=\left(\begin{array}{c}
\frac{1}{2}\left(\left|x_{s}\right|^{2}+1\right) \mathrm{e}^{-t_{s}}+\frac{\mathrm{e}^{t_{s}}}{2} \\
\frac{\left|x_{s}\right|^{2}-1}{2} \mathrm{e}^{-t_{s}}+\frac{\mathrm{e}^{t_{s}}}{2} \\
\mathrm{e}^{-t_{s}} x_{s}
\end{array}\right)
$$

On the other hand,

$$
\mathbf{g}_{s}^{0}=\left(\operatorname{ch} \rho_{s}\right) \varepsilon_{0}+\left(\operatorname{sh} \rho_{s}\right) \sigma_{s}
$$

Comparing these two expressions, we obtain

$$
\sigma_{\infty}=\lim _{s \rightarrow+\infty} \frac{\left|x_{s}\right|^{2}-1+\mathrm{e}^{2 t_{s}}}{\left|x_{s}\right|^{2}+1+\mathrm{e}^{2 t_{s}}} \varepsilon_{1}+\frac{2 x_{s}}{\left|x_{s}\right|^{2}+1+\mathrm{e}^{2 t_{s}}}=\frac{\left|x_{\infty}\right|^{2}-1}{\left|x_{\infty}\right|^{2}+1} \varepsilon_{1}+\frac{2 x_{\infty}}{\left|x_{\infty}\right|^{2}+1} .
$$

This is the analytical expression of the stereographic projection of $x_{\infty} \in \mathbb{R}^{d-1} \subset\left\langle\varepsilon_{2}, \ldots, \varepsilon_{d}\right\rangle$ on $\mathbb{S}^{d-1} \subset\left\langle\varepsilon_{1}, \ldots, \varepsilon_{d}\right\rangle$. 
2. We are now going to prove that $\lim _{s \rightarrow+\infty} q\left(\xi_{s}, \varepsilon_{0}+\sigma_{\infty}\right)=\frac{r_{\infty}}{1+\left|x_{\infty}\right|^{2}}$. We shall consider $\mathbb{S}^{d-1}$ as a subset of $\left\langle\varepsilon_{1}, \ldots, \varepsilon_{d}\right\rangle$ and identify $\mathbb{R}^{d-1}$ to $\left\langle\varepsilon_{2}, \ldots, \varepsilon_{d}\right\rangle$. We use the notation $\langle.,$.$\rangle for the Euclidean scalar product in \mathbb{R}^{1+d}$.

a) $\bullet$ We first give in the forthcoming identity (5.6) an expression of $r_{n}$ involving only $x_{n} \in \mathbb{R}^{d-1}$ and $\xi_{n} \in \mathbb{R}^{1, d}$. Use the fact that $N\left(x_{n}\right)\left(\varepsilon_{0}+\varepsilon_{1}\right)=\varepsilon_{0}+\varepsilon_{1}$ to re-write the decomposition

$$
\begin{aligned}
\mathbf{e}_{n} & =\left(\xi_{n}, \mathbf{g}_{n}\right)=\left(\xi_{n}, N\left(x_{n}\right) A\left(t_{n}\right) K_{n}\right) \\
& =\left(\left\langle N\left(x_{n}\right)^{-1} \xi_{n}, \varepsilon_{0}+\varepsilon_{1}\right\rangle\left(\varepsilon_{0}+\varepsilon_{1}\right), N\left(x_{n}\right)\right)\left(N\left(-x_{n}\right) \xi_{n}-\left\langle N\left(-x_{n}\right) \xi_{n}, \varepsilon_{0}+\varepsilon_{1}\right\rangle\left(\varepsilon_{0}+\varepsilon_{1}\right), A\left(t_{n}\right) K_{n}\right),
\end{aligned}
$$

which proves that the $\mathcal{D}^{-}$-component of $\mathbf{e}_{n}$ is

$$
\left(\left\langle N\left(-x_{n}\right) \xi_{n}, \varepsilon_{0}+\varepsilon_{1}\right\rangle\left(\varepsilon_{0}+\varepsilon_{1}\right), N\left(x_{n}\right)\right) .
$$

Since

$$
{ }^{t} N\left(x_{n}\right)^{-1}\left(\varepsilon_{0}+\varepsilon_{1}\right)=\left(\left|x_{n}\right|^{2}+1\right) \varepsilon_{0}+\left(1-\left|x_{n}\right|^{2}\right) \varepsilon_{1}-2 x_{n},
$$

we obtain

$$
\begin{aligned}
r_{n} & =\left\langle\xi_{n},{ }^{t} N\left(x_{n}\right)^{-1}\left(\varepsilon_{0}+\varepsilon_{1}\right)\right\rangle=\left(\left|x_{n}\right|^{2}+1\right)\left\langle\varepsilon_{0}-\left(\frac{\left|x_{n}\right|^{2}-1}{\left|x_{n}\right|^{2}+1} \varepsilon_{1}+\frac{2 x_{n}}{\left|x_{n}\right|^{2}+1}\right), \xi_{n}\right\rangle \\
& =\left(\left|x_{n}\right|^{2}+1\right) q\left(\varepsilon_{0}+\left(\frac{\left|x_{n}\right|^{2}-1}{\left|x_{n}\right|^{2}+1} \varepsilon_{1}+\frac{2 x_{n}}{\left|x_{n}\right|^{2}+1}\right), \xi_{n}\right) .
\end{aligned}
$$

Remark that

$$
\left(\operatorname{th} \rho_{s}\right) \sigma_{s}=\frac{\left|x_{s}\right|^{2}-1+\mathrm{e}^{2 t_{s}}}{\left|x_{s}\right|^{2}+1+\mathrm{e}^{2 t_{s}}} \varepsilon_{1}+\frac{2 x_{s}}{\left|x_{s}\right|^{2}+1+\mathrm{e}^{2 t_{s}}},
$$

is almost equal to $\left(\frac{\left|x_{s}\right|^{2}-1}{\left|x_{s}\right|^{2}+1} \varepsilon_{1}+\frac{2 x_{s}}{\left|x_{s}\right|^{2}+1}\right)$.

- We now show that $q\left(\varepsilon_{0}+\left(\frac{\left|x_{n}\right|^{2}-1}{\left|x_{n}\right|^{2}+1} \varepsilon_{1}+\frac{2 x_{n}}{\left|x_{n}\right|^{2}+1}\right), \xi_{n}\right)$ and $q\left(\varepsilon_{0}+\sigma_{n}, \xi_{n}\right)$ have the same limit. We can do this in two steps:

1. $q\left(\varepsilon_{0}+\left(\frac{\left|x_{n}\right|^{2}-1}{\left|x_{n}\right|^{2}+1} \varepsilon_{1}+\frac{2 x_{n}}{\left|x_{n}\right|^{2}+1}\right), \xi_{n}\right)-q\left(\varepsilon_{0}+\left(\operatorname{th} \rho_{n}\right) \sigma_{n}, \xi_{n}\right)=o(1)$,

2. $q\left(\varepsilon_{0}+\left(\operatorname{th} \rho_{n}\right) \sigma_{n}, \xi_{n}\right)-q\left(\varepsilon_{0}+\sigma_{n}, \xi_{n}\right)=o(1)$.

1 is a consequence of the following easily established estimates:

- $\forall \varepsilon>0,\left|\xi_{n}\right| \leqslant O\left(\mathrm{e}^{(1+\varepsilon) \frac{d-1}{2} n}\right)^{26}$,

- $\left|\frac{\left|x_{n}\right|^{2}-1+\mathrm{e}^{2 t_{n}}}{\left|x_{n}\right|^{2}+1+\mathrm{e}^{2 t_{n}}}-\frac{\left|x_{n}\right|^{2}-1}{\left|x_{n}\right|^{2}+1}\right|=O\left(\mathrm{e}^{2 t_{n}}\right)$,

- $\left|\frac{1}{\left|x_{n}\right|^{2}+1+\mathrm{e}^{2 t_{n}}}-\frac{1}{\left|x_{n}\right|^{2}+1}\right|=O\left(\mathrm{e}^{2 t_{n}}\right)$.

2 since

$$
q\left(\varepsilon_{0}+\left(\operatorname{th} \rho_{n}\right) \sigma_{n}, \xi_{n}\right)-q\left(\varepsilon_{0}+\sigma_{n}, \xi_{n}\right)=q\left(\left(1-\operatorname{th} \rho_{n}\right) \sigma_{n}, \xi_{n}\right) \leqslant O\left(\mathrm{e}^{-2 \rho_{n}}\right) O\left(\mathrm{e}^{(1+\varepsilon) \frac{d-1}{2} n}\right),
$$

\footnotetext{
${ }^{26}\left|\xi_{n}\right|$ is the $(d-1)$-Euclidean norm of $\xi_{s} \in \mathbb{R}^{1+d}$.
} 
the second point is a direct consequence of the estimate $\frac{\rho_{n}}{n} \rightarrow \frac{d-1}{2}$ of Proposition 2.5. As a consequence, we have

$$
\left(1+\left|x_{n}\right|^{2}\right) q\left(\varepsilon_{0}+\sigma_{n}, \xi_{n}\right) \rightarrow r_{\infty}
$$

b) It remains to prove that $q\left(\left(1, \sigma_{n}\right), \xi_{n}\right)$ and $q\left(\left(1, \sigma_{\infty}\right), \xi_{n}\right)$ have the same limit.

Denote by (.,.) Euclidean scalar product in $\mathbb{R}^{d}$. For $\sigma \in \mathbb{S}^{d-1}$,

$$
\begin{aligned}
q\left(\varepsilon_{0}+\sigma, \xi_{n}\right) & =q\left(\varepsilon_{0}+\sigma, \xi_{0}\right)+\int_{0}^{n} \operatorname{ch} \rho_{s} \mathrm{~d} s-\int_{0}^{n}\left(\operatorname{sh} \rho_{s}\right)\left(\sigma_{s}, \sigma\right) \mathrm{d} s \\
& =q\left(\varepsilon_{0}+\sigma, \xi_{0}\right)+\int_{0}^{n}\left(\operatorname{ch} \rho_{s}-\operatorname{sh} \rho_{s}\right) \mathrm{d} s+\int_{0}^{n}\left(\operatorname{sh} \rho_{s}\right)\left(1-\left(\sigma_{s}, \sigma\right)\right) \mathrm{d} s .
\end{aligned}
$$

So,

$$
\begin{aligned}
q\left(\varepsilon_{0}+\sigma_{n}, \xi_{n}\right)-q\left(\varepsilon_{0}+\sigma_{\infty}, \xi_{n}\right) & =q\left(\varepsilon_{0}+\sigma_{n}, \xi_{0}\right)-q\left(\varepsilon_{0}+\sigma_{\infty}, \xi_{0}\right) \\
& +\int_{0}^{n}\left(\operatorname{sh} \rho_{s}\right)\left\{\left(1-\left(\sigma_{s}, \sigma_{n}\right)\right)-\left(1-\left(\sigma_{s}, \sigma_{\infty}\right)\right)\right\} \mathrm{d} s \\
& =o(1)+\int_{0}^{\infty}\left(\operatorname{sh} \rho_{s}\right)\left\{\left(1-\left(\sigma_{s}, \sigma_{n}\right)\right)-\left(1-\left(\sigma_{s}, \sigma_{\infty}\right)\right)\right\} \mathbf{1}_{s \leqslant n} \mathrm{~d} s
\end{aligned}
$$

We want to apply the dominated convergence theorem to show that the integral tends to 0 . In order to do this, we need to majorize $\left\{\left(1-\left(\sigma_{s}, \sigma_{n}\right)\right)-\left(1-\left(\sigma_{s}, \sigma_{\infty}\right)\right)\right\} \mathbf{1}_{s \leqslant n}$ by some function $f(s)$ independent of $n$ and such that $\int_{0}^{\infty}\left(\operatorname{sh} \rho_{s}\right) f(s) \mathrm{d} s<\infty$.

Denote by $d_{s, n}$ the spherical distance between $\sigma_{s}$ and $\sigma_{n}$, and $d_{s, \infty}$ the spherical distance between $\sigma_{s}$ and $\sigma_{\infty}$. Since

$$
\begin{aligned}
& 1-\left(\sigma_{s}, \sigma_{n}\right)=\frac{d_{s, n}^{2}}{2}+o\left(d_{s, n}^{2}\right), \\
& 1-\left(\sigma_{s}, \sigma_{n}\right)=\frac{d_{s, \infty}^{2}}{2}+o\left(d_{s, \infty}^{2}\right),
\end{aligned}
$$

we estimate $d_{s, n}$ and $d_{s, \infty}$.

As $\sigma_{s}=\Sigma\left(T_{s}\right)$, where $\Sigma$ is a Brownian motion on $\mathbb{S}^{d-1}$ and $T_{s}$ a converging random time change independent of $\Sigma$, we can use estimates on the continuity modulus of $\Sigma$ to majorize $d_{s, n}$ and $d_{s, \infty}$. In their article [5], Baldi and Chaleyat-Maurel showed that Levy's estimate on the continuity modulus of real Brownian motion has an analogous for elliptic diffusions, provided one replaces Euclidean geometry of $\mathbb{R}$ by the Riemannian geometry associated with the diffusion. For Brownian motion $\Sigma$ on $\mathbb{S}^{d-1}$, this geometry is the usual geometry induced by the ambient Euclidean space. So, if $\operatorname{osc}(\Sigma ; u, v)$ denotes the oscillation of $\Sigma$ on the time interval $[u, v]$, one has almost surely

$$
\operatorname{osc}(\Sigma ; u, v) \leqslant \sqrt{3(v-u) \log \frac{1}{v-u}},
$$

provided $(v-u)$ is small enough (and $[u, v]$ is in a fixed interval). Since

$$
d_{s, n} \text { and } d_{s, \infty} \leqslant \operatorname{osc}\left(\Sigma ; T_{s}, T_{\infty}\right)
$$

we have

$$
d_{d, n}^{2}+d_{s, \infty}^{2} \leqslant 2 \operatorname{osc}\left(\Sigma ; T_{s}, T_{\infty}\right)^{2} \leqslant 6\left(T_{\infty}-T_{s}\right) \log \frac{1}{T_{\infty}-T_{s}}
$$


for $s$ large enough.

$$
\begin{gathered}
\text { As } \left.T_{\infty}-T_{s}=\int_{s}^{\infty} \frac{\mathrm{d} r}{\operatorname{sh}^{2} \rho_{r}} \leqslant \int_{s}^{\infty} \frac{4 \mathrm{~d} r}{\left(\mathrm{e}^{\rho_{r}}-1\right)^{2}}, \text { and the function } \varepsilon \mapsto \varepsilon \log \frac{1}{\varepsilon} \text { increases on }\right] 0, \frac{1}{e}[, \\
d_{d, n}^{2}+d_{s, \infty}^{2} \leqslant 24\left(\int_{s}^{\infty} \frac{\mathrm{d} r}{\left(\mathrm{e}^{\rho_{r}}-1\right)^{2}}\right) \log \left(\int_{s}^{\infty} \frac{\mathrm{d} r}{\left(\mathrm{e}^{\rho_{r}}-1\right)^{2}}\right)^{-1},
\end{gathered}
$$

for $s$ large enough.

Let $\varepsilon>0$. We saw in Proposition 2.5 that the inequalities $\frac{d-1}{2+\varepsilon} r \leqslant \rho_{r} \leqslant \frac{d-1}{2-\varepsilon} r$, hold for $r$ large enough; so, a majoration of the form

$$
d_{d, n}^{2}+d_{s, \infty}^{2} \leqslant C \mathrm{e}^{-2 \frac{d-1}{2+\frac{s}{2}} s},
$$

holds for $s$ large enough. It follows from (5.9) that the same kind of majoration holds for

$$
\left\{\left(1-\left(\sigma_{s}, \sigma_{n}\right)\right)-\left(1-\left(\sigma_{s}, \sigma_{\infty}\right)\right)\right\} \mathbf{1}_{s \leqslant n},
$$

independently of $n$, for $s$ large. As we eventually have $\operatorname{sh} \rho_{s} \leqslant \mathrm{e}^{\frac{d-1}{2-\varepsilon} s}$,

$$
\left(\operatorname{sh} \rho_{s}\right)\left\{\left(1-\left(\sigma_{s}, \sigma_{n}\right)\right)-\left(1-\left(\sigma_{s}, \sigma_{\infty}\right)\right)\right\} \mathbf{1}_{s \leqslant n} \leqslant C \exp \left(-(d-1)\left(\frac{2}{2+\frac{\varepsilon}{2}}-\frac{1}{2-\varepsilon}\right) s\right) .
$$

The majorant being integrable for $\varepsilon>0$ small enough, we can use the dominated convergence theorem in (5.8), and conclude that

$$
q\left(\varepsilon_{0}+\sigma_{n}, \xi_{n}\right)-q\left(\varepsilon_{0}+\sigma_{\infty}, \xi_{n}\right)=o(1) .
$$

Now, (5.6), (5.7) and (5.10) prove that

$$
\lim _{n \rightarrow+\infty} q\left(\varepsilon_{0}+\sigma_{\infty}, \xi_{n}\right)=\frac{r_{\infty}}{\left|x_{\infty}\right|^{2}+1} .
$$

It remains to remark that the function $s \mapsto q\left(\varepsilon_{0}+\sigma_{\infty}, \xi_{s}\right)$ increases to finally obtain

$$
\lim _{s \rightarrow+\infty} q\left(\varepsilon_{0}+\sigma_{\infty}, \xi_{s}\right)=\frac{r_{\infty}}{\left|x_{\infty}\right|^{2}+1} .
$$

\subsection{Proof of Theorem 4.9 on causal boundary of $\mathbb{R}^{1, d}$}

Recall that $\left(\widetilde{\varepsilon}_{0}, \ldots, \widetilde{\varepsilon}_{2+d}\right)$ is the canonical basis of $\mathbb{R}^{2,1+d}$, that $\left(\varepsilon_{0}, \ldots, \varepsilon_{n-1}\right)$ is the canonical basis of $\mathbb{R}^{1, d}$, and that the map $i: \mathbb{R}^{1, d} \rightarrow\left\langle\widetilde{\varepsilon}_{1}, \ldots, \widetilde{\varepsilon}_{1+d}\right\rangle$ is the linear identification of $\mathbb{R}^{1, d}$ with $\left\langle\widetilde{\varepsilon}_{1}, \ldots, \widetilde{\varepsilon}_{n}\right\rangle$ sending $\varepsilon_{j}$ on $\widetilde{\varepsilon}_{j+1}$.

First, we show that the lightlike geodesics of Einstein universe have the simple description given in Proposition 4.6. The proof makes use of the conformal embedding of $\mathbb{R}^{1, d}$ in $\mathbf{E i n}_{1+d}$ as a dense subset, with boundary C. We identify $\mathbb{R}^{1, d}$ with its stereographic projection.

Proposition 5.5. The lightlike geodesics of $\mathbf{E i n}_{1+d}$ are the projections in $\mathbf{E i n}_{1+d}$ of totally isotrope planes of $\mathbb{R}^{2,1+d}$.

Proof of Proposition 5.5. Using the transitive action of $P O(2,1+d)$ on the bundle of lightlike geodesic over $\operatorname{Ein}_{1+d}$, we are brought back to show that the lightlike geodesic $\gamma: t \in \mathbb{R} \mapsto t\left(\varepsilon_{0}+\varepsilon_{1}\right)$, (locally) embeds into the projection of a totally degenerated plane of $\mathbb{R}^{2,1+d}$. We see on formula (4.2) defining $j$ that this is indeed the case: $j(\gamma(\mathbb{R}))=\pi\left(\left\langle\widetilde{\varepsilon}_{d+2}, \widetilde{\varepsilon}_{1}+\widetilde{\varepsilon}_{2}\right\rangle\right)$. 
Proof of Theorem 4.9. Given $v \in \mathbb{R}^{1, d}$, define the geodesic $\gamma_{s}=a+s v \in \mathbb{R}^{1, d}, s \in \mathbb{R}$. The image of $\gamma$ by $j$ is the curve of $C^{2,1+d}$

$$
j\left(\gamma_{s}\right)=s^{2} q(v) \widetilde{\varepsilon}_{0}+2 s\left(q(a, v) \widetilde{\varepsilon}_{0}+i(v)\right)+2 i(a)+q(a) \widetilde{\varepsilon}_{0}+\widetilde{\varepsilon}_{d+2} .
$$

1. We first show that if $v$ is not a null vector $\gamma$ converges to $\mathbf{p}$. For such a vector $v, q(v) \neq 0$ and

$$
\begin{aligned}
\lim _{s \rightarrow+\infty} \pi\left(j\left(\gamma_{s}\right)\right) & =\lim _{s \rightarrow+\infty} \pi\left(s^{2} q(v) \widetilde{\varepsilon}_{0}+2 s\left(q(a, v) \widetilde{\varepsilon}_{0}+i(v)\right)+2 i(a)+q(a) \widetilde{\varepsilon}_{0}+\widetilde{\varepsilon}_{d+2}\right) \\
& =\lim _{s \rightarrow+\infty} \pi\left(\widetilde{\varepsilon}_{0}+o\left(s^{-1}\right)\right)=\pi\left(\widetilde{\varepsilon}_{0}\right)=\mathbf{p}
\end{aligned}
$$

2. We now describe what happens to lightlike geodesics of $\mathbb{R}^{1, d}$. We just need two ingredients to obtain the description given in point 2 of Theorem 4.9. The first one is that for a null vector $v \in \mathbb{R}^{1, d}$ and an associated lightlike geodesic $\gamma_{s}=a+s v$,

$$
\lim _{s \rightarrow+\infty} \pi\left(j\left(\gamma_{s}\right)\right)=\pi\left(q(a, v) \widetilde{\varepsilon}_{0}+i(v)\right) .
$$

The other one is the remark that $\mathbf{C} \backslash\{\mathbf{p}\}$ is foliated by lightlike geodesics ${ }^{27}$ :

$$
\mathbf{C} \backslash\{\mathbf{p}\}=\coprod_{\sigma \in \mathbb{S}^{d-1}} \pi\left(\mathbb{R} \widetilde{\varepsilon}_{0}+i\left(\varepsilon_{0}+\sigma\right)\right)=\coprod_{\sigma \in \mathbb{S}^{d-1}} \Delta_{\sigma} \backslash\{\mathbf{p}\} .
$$

All the results stated in point 2 can be read directly on formula (5.13).

3. Parameterization of $\mathbf{C} \backslash\{\mathbf{p}\} \bullet$ Recall $p_{\sigma}(\ell)$ is the limit of any lightlike geodesic $\left\{a+t\left(\varepsilon_{0}+\sigma\right)\right\}_{t \in \mathbb{R}}$ such that $q\left(a, \varepsilon_{0}+\sigma\right)=\ell$. The injectivity of $(\sigma, \ell) \in \mathbb{S}^{d-1} \mathbb{R} \mapsto p_{\sigma}(\ell) \in \mathbf{C} \backslash\{\mathbf{p}\}$ reads directly on formula (5.13). To prove that it is a diffeomorphism, define

$$
\varphi: \mathbb{S}^{d-1} \rightarrow \mathbf{C} \backslash\{\mathbf{p}\}, \sigma \mapsto p_{\sigma}(0)=\pi\left(i\left(\varepsilon_{0}+\sigma\right)\right) ;
$$

$\varphi$ is easily seen to be a diffeomorphism from $\mathbb{S}^{d-1}$ to $\varphi\left(\mathbb{S}^{d-1}\right)$.

Introduce the family $\varphi(t,$.$) of translations of \mathbb{R}^{1, d}$

$$
\varphi(t, .): \mathbb{R}^{1, d} \rightarrow \mathbb{R}^{1, d}, \zeta \mapsto \zeta+t \varepsilon_{0} .
$$

The element of $O(2,1+d)$ whose action on $\operatorname{Ein}_{1+d}$ induces $\varphi(t,$.$) on \mathbb{R}^{1, d} \subset \mathbf{E i n}_{1+d}$ has $\operatorname{matrix}^{28}$

$$
\left(\begin{array}{cccccc}
1 & t & 0 & \cdots & 0 & t^{2} \\
& 1 & 0 & \cdots & 0 & 2 t \\
& & \ddots & & \vdots & 0 \\
& & & \ddots & 0 & \vdots \\
& (0) & & & 1 & 0 \\
& & & & & 1
\end{array}\right) .
$$

We see on this expression that one can extend this smooth flow of translations to a smooth flow of transforms of $P O(2,1+d)$ acting on $\operatorname{Ein}_{1+d}$, say $\phi(t,$.$) .$

\footnotetext{
${ }^{27}$ This comes from the description of the lightlike geodesics of $\mathbf{E i n}_{1+d}$ and from the definition of $\mathbf{C}$.

28 This matrix belongs to $O(2,1+d)$. Check that it has the good action on $\mathbb{R}^{1, d}$, i.e. look at coordinates 1 to $1+d$. As Liouville theorem asserts the existence of a unique such transform, this one is the good one.
} 
Lemma 5.6. The flow $\{\phi(t, .)\}_{t \in \mathbb{R}}$ preserves each lightlike geodesic of $\mathbf{C}$, and is transitive on each $\Delta_{\sigma} \backslash\{\mathbf{p}\}$.

Proof of Lemma 5.6. Since a point $p_{\sigma}(\ell)$ is the limit of a lightlike geodesic $\left\{\gamma_{s}\right\}_{s \geqslant 0}=\left\{a+s\left(\varepsilon_{0}+\sigma\right)\right\}_{s \geqslant 0}$, with $\ell=q\left(a, \varepsilon_{0}+\sigma\right)$

$$
\phi\left(t, p_{\sigma}(\ell)\right)=\lim _{s \rightarrow+\infty} \phi\left(t, \gamma_{s}\right) .
$$

As $\phi\left(t, \gamma_{s}\right)=\pi \circ j\left(\gamma_{s}+t \varepsilon_{0}\right)$, the formulas for $j$ and $\pi$ give

$$
\begin{aligned}
\phi\left(t, p_{\sigma}(\ell)\right) & =\lim _{s \rightarrow+\infty} \phi\left(t, \gamma_{s}\right)=\pi\left(q\left(a+t \varepsilon_{0}, \varepsilon_{0}+\sigma\right) \varepsilon_{0}+i\left(\varepsilon_{0}+\sigma\right)\right) \\
& =\pi\left((\ell+t) \varepsilon_{0}+i\left(\varepsilon_{0}+\sigma\right)\right)=p_{\sigma}(\ell+t) .
\end{aligned}
$$

So, the flow preserves each geodesic $\Delta_{\sigma}$ of $\mathbf{C}$; transitiveness of the action reads on formulas (5.15) and (5.14).

Since $\varphi(\sigma)=p_{\sigma}(0)$, formula $(5.15)$ reads

$$
\phi(\ell, \sigma)=p_{\sigma}(\ell) .
$$

The flow property of $\phi$ and the fact that $\sigma \mapsto p_{\sigma}(0)$ is a diffeomorphism prove that the application $(\sigma, \ell) \mapsto p_{\sigma}(\ell)$ is indeed a parameterization of $\mathbf{C} \backslash\{\mathbf{p}\}$.

4. Causal boundary of $\mathbb{R}^{1, d} \bullet$ To prepare the proof of point 4 we need an intermediate result describing the past of a point $p_{\sigma}(\ell)$ in $\mathbb{R}^{1, d}$. A point $\xi \in \mathbb{R}^{1, d}$ being given, define its lightcone as the set

$$
\mathcal{C}^{0}(\xi)=\left\{\zeta \in \mathbb{R}^{1, d} ; q(\zeta-\xi)=0\right\},
$$

and its future as

$$
\mathcal{C}^{\geqslant 0}(\xi)=\left\{\zeta \in \mathbb{R}^{1, d} ; q(\zeta-\xi) \geqslant 0, \zeta^{0}-\xi^{0} \geqslant 0\right\} .
$$

These are subsets of $\mathbf{E i n}_{1+d}$. Future cones and null cones are linked by the relation

$$
\overline{\mathcal{C} \geqslant 0}(\xi)=\overline{\bigcup_{t \geqslant 0} \overline{\mathcal{C}^{0}\left(\xi+t \varepsilon_{0}\right)}}
$$

It is a direct consequence of the result of point 3 that

- $\overline{\mathcal{C}^{0}(\xi)} \cap \mathbf{C}=\left\{p_{\sigma}\left(q\left(\varepsilon_{0}+\sigma, \xi\right)\right) ; \sigma \in \mathbb{S}^{d-1}\right\}$ is diffeomorphic to $\mathbb{S}^{d-1}$,

- $\overline{\mathcal{C} \geqslant 0}(\xi) \cap(\mathbf{C} \backslash\{\mathbf{p}\})=\left\{p_{\sigma}(\ell) ; \ell \geqslant q\left(\varepsilon_{0}+\sigma, \xi\right)\right\}$.

Define now

$$
\text { Past }\left(p_{\sigma}(\ell), \mathbb{R}^{1, d}\right)=\left\{\zeta \in \mathbb{R}^{1, d} ; \exists \gamma: \mathbb{R}^{\geqslant 0} \rightarrow \mathbb{R}^{1, d} \text {, is future-oriented, causal, and } \gamma_{0}=\zeta, \lim _{s \rightarrow+\infty} \gamma_{s}=p_{\sigma}(\ell)\right\} .
$$

It is straightforward that one can also write Past $\left(p_{\sigma}(\ell), \mathbb{R}^{1, d}\right)$ as

$$
\left\{\zeta \in \mathbb{R}^{1, d} ; p_{\sigma}(\ell) \in \overline{\mathcal{C} \geqslant 0}(\zeta)\right\} .
$$

For example, $\operatorname{Past}\left(\mathbf{p}, \mathbb{R}^{1, d}\right)=\mathbb{R}^{1, d}$.

Remark. Let us insist on the fact that we look at the past of $p_{\sigma}(\ell)$ in $\mathbb{R}^{1, d}$ : the past of $p_{\sigma}(\ell)$ is Einstein universe is the whole space. Indeed, let us prove this fact for the point 0 ; the homogeneity of the space confering the same property to all the other points.

Choose $\sigma \in \mathbb{S}^{d-1}$ and call $\Gamma: \mathbb{R} \rightarrow \mathbb{R}^{1, d} \subset \mathbf{E i n}_{1+d}$ the geodesic $s \mapsto s\left(\varepsilon_{0}+\sigma\right)$. One reads on formula (5.12) that $\Gamma(s)$ converges towards $p_{\sigma}(0)$ as $s \rightarrow+\infty$ and $s \rightarrow-\infty$. This implies that the half-geodesic $\Gamma((-\infty, 0])$ is 
in the future of $p_{\sigma}(0)$ and, as such, is also in the future of 0 ; the point 0 is thus in its own future and in its own past.

Lemma 5.7. Past $\left(p_{\sigma}(\ell), \mathbb{R}^{1, d}\right)=\left\{\zeta \in \mathbb{R}^{1, d} ; q\left(\zeta, \varepsilon_{0}+\sigma\right) \leqslant \ell\right\}$.

$$
\overline{\operatorname{Past}\left(p_{\sigma}(\ell), \mathbb{R}^{1, d}\right)} \cap \mathbf{C}=\left\{p_{\sigma}(r),, r \leqslant \ell\right\} .
$$

Proof. We show the first point, the second being a direct consequence of it. Given $\zeta_{0} \in \mathbb{R}^{1, d}$ with $q\left(\zeta_{0}, \varepsilon_{0}+\sigma\right) \leqslant \ell$, find a timelike path from $\zeta_{0}$ to the hyperplane $\left\{q\left(., \varepsilon_{0}+\sigma\right)=\ell\right\}$. It hits the hyperplane at $\zeta_{1}$. Then, follow the unique lightlike geodesic with direction $\sigma$, passing through $\zeta_{1}$. The concatenation of the two paths is a causal path from $\zeta$ to $p_{\sigma}(\ell)$. So, $\left\{q\left(., \varepsilon_{0}+\sigma\right) \leqslant \ell\right\} \subset \operatorname{Past}\left(p_{\sigma}(\ell), \mathbb{R}^{1, d}\right)$.

On the other side, if $q\left(\zeta_{0}, \varepsilon_{0}+\sigma\right)>\ell$, then $p_{\sigma}(\ell)$ cannot be in $\overline{\mathcal{C} \geqslant 0\left(\zeta_{0}\right) \cap(\mathbf{C} \backslash\{\mathbf{p}\})}$, since

$$
\overline{\mathcal{C} \geqslant 0\left(\zeta_{0}\right) \cap(\mathbf{C} \backslash\{\mathbf{p}\})}=\left\{p_{\underline{\sigma}}(\underline{\ell}) ; \underline{\ell} \geqslant q\left(\varepsilon_{0}+\underline{\sigma}, \zeta_{0}\right)\right\} .
$$

4(a). The proof of the point 4(a) relies on an algebraic lemma.

Lemma 5.8. $P O(2,1+d)$ acts transitively on the pairs of points of $\mathbf{E i n}_{1+d}$ that are not on the same lightlike geodesic.

Proof. Since any pair of points of $\mathbf{E i n}_{1+d}$ that are not on the same lightlike geodesic is of the form $(\pi(u), \pi(v))$, with $u$ and $v$ null vectors, not orthogonal, we are brought back to show that $O(2,1+d)$ acts transitively on the set of such pairs $(u, v)$, that is, $O(2,1+d)$ acts transitively on the set of planes of signature $(+,-)$. This is a well known (elementary) fact.

Given $\xi \in \mathbb{R}^{1, d}$, define $\mathcal{C}^{>0}(\xi)$ as the inside of the future $\mathcal{C}^{\geqslant 0}(\xi)$ of $\xi$, and for $\zeta$ in Einstein universe, define $\mathcal{C}^{<0}(\zeta)$ as the inside of its past $\operatorname{Past}\left(\zeta, \mathbb{R}^{1, d}\right)$ in $\mathbb{R}^{1, d}$. For example, since $\operatorname{Past}\left(\mathbf{p}, \mathbb{R}^{1, d}\right)=\mathbb{R}^{1, d}$, any timelike path $\gamma$ is in $\mathcal{C}^{<0}(\mathbf{p})$.

Let $\left\{\gamma_{s}\right\}_{s \geqslant 0}$ be a timelike path in $\mathbb{R}^{1, d}$, future-oriented and inextensible. The points $\gamma_{0}$ and $\mathbf{p}$ not being on the same lightlike geodesic of $\mathbf{E i n}_{1+d}$, we can find an element $T$ of $P O(2,1+d)$ that maps $\gamma_{0}$ and $\mathbf{p}$ to $-\varepsilon_{0}$ and $\varepsilon_{0}$ respectively. We prove the statement 4 (a) for the path $T \circ \gamma$, with $T(\mathbf{C})$ instead of $\mathbf{C}$; this implies the original statement. We shall still denote by $\gamma$ the path $T \circ \gamma$. The path $\gamma$ being timelike it remains in the diamond $\mathcal{D} \equiv \mathcal{C}^{>0}\left(-\varepsilon_{0}\right) \cap \mathcal{C}^{<0}\left(\varepsilon_{0}\right) \subset \mathbb{R}^{1, d}$.

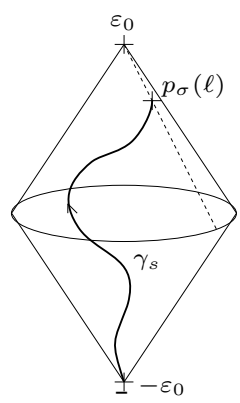

The following lemma is an easily proved and useful fact.

Lemma 5.9. The family $\left\{\mathcal{C}^{>0}\left(m-t \varepsilon_{0}\right) \cap \mathcal{C}^{<0}\left(m+t \varepsilon_{0}\right) ; m \in \mathbb{R}^{1, d}, t>0\right\}$ is a basis of the topology of $\mathbb{R}^{1, d 29}$.

Definition 5.10. We call a neighbourhood of $m$ of the form $\mathcal{C} \geqslant 0\left(m-t \varepsilon_{0}\right) \cap \mathcal{C} \leqslant 0\left(m+t \varepsilon_{0}\right)$ a diamond neighbourhood of $m$, written $\mathcal{D}(m, t)$.

\footnotetext{
${ }^{29}$ The statement is true in a much greater generality.
} 
Denote by $C l(\gamma)$ the set of points of the past lightcone of $\varepsilon_{0}$ that are in the closure of $\gamma$, and suppose that $C l(\gamma)$ contains two distinct points $p_{1}$ and $p_{2}$. Let $\mathcal{D}\left(p_{1}, t_{1}\right)$ and $\mathcal{D}\left(p_{2}, t_{2}\right)$ two diamond neighbourhoods of $p_{1}$ and $p_{2}$, respectively, that do not intersect.

Since $\gamma$ is a timelike path, and $p_{1} \in C l(\gamma)$, for any $s \geqslant 0$, the point $\gamma_{s} \in \mathcal{C}^{<0}\left(p_{1}\right)$. All the more, since $p_{1} \in C l(\gamma)$, the path $\gamma$ enters $\mathcal{D}\left(p_{1}, t_{1}\right)$ at some time; in particular, there is a time $s_{1}$ such that $\gamma_{s_{1}} \in \mathcal{C}>0\left(p_{1}-\right.$ $\left.t_{1} \varepsilon_{0}\right)$. No timelike path started from a point of $\mathcal{C}^{>0}\left(p_{1}-t_{1} \varepsilon_{0}\right)$ can exit from this set. So, $\gamma$ remains in $\mathcal{C}^{>0}\left(p_{1}-t_{1} \varepsilon_{0}\right) \cap \mathcal{C}^{\leqslant 0}\left(p_{1}\right) \subset D\left(p_{1}, t_{1}\right)$, from that time on. But since $\mathcal{D}\left(p_{1}, t_{1}\right)$ and $\mathcal{D}\left(p_{2}, t_{2}\right)$ do not intersect, $p_{2}$ cannot belong to $C l(\gamma)$, contradicting the hypothesis.

We denote by $\gamma_{\infty}$ the unique point of $\mathbf{C}$ in $C l(\gamma)$.

4(b). i) Let $\sigma \in \mathbb{S}^{d-1}$ and $\ell \in \mathbb{R}$ be given, and let $\left\{\gamma_{s}\right\}_{s \geqslant 0}$ be a timelike path such that $q\left(\gamma_{s}, \varepsilon_{0}+\sigma\right) \underset{s \rightarrow+\infty}{\longrightarrow} \ell$.

As $\left\{\gamma_{s}\right\}_{s \geqslant 0}$ is timelike, the function $s \in \mathbb{R} \geqslant 0 \rightarrow q\left(\gamma_{s}, \varepsilon_{0}+\sigma\right)$ increases. We know by Lemma 5.7 that the point $\gamma_{s}$ belongs to Past $\left(p_{\sigma}(\ell), \mathbb{R}^{1, d}\right)$, and that $\gamma_{\infty} \in\left\{p_{\sigma}(r) ; r \leqslant \ell\right\}$.

Should $\gamma_{\infty}$ be equal to some $p_{\sigma}(r)$, with $r<\ell$, then $q\left(\gamma_{s}, \varepsilon_{0}+\sigma\right)$ would remain $\leqslant r$, contradicting the hypothesis. This shows that $\gamma_{\infty}=p_{\sigma}(\ell)$.

ii) Reciprocally, suppose that $\gamma_{\infty}=p_{\sigma}(\ell)$, for some $\sigma \in \mathbb{S}^{d-1}$ and $\ell \in \mathbb{R}$. Then $q\left(\gamma_{s}, \varepsilon_{0}+\sigma\right) \leqslant \ell$, for all $s \geqslant 0$. This function of $s$ increases to a limit $r \leqslant \ell$. Point $i)$ shows that $\gamma_{\infty}=p_{\sigma}(r)$, so $r=\ell$. Thus, given $\sigma \in \mathbb{S}^{d-1}$ and $\ell \in \mathbb{R}$

$$
\gamma_{s} \underset{s \rightarrow+\infty}{\longrightarrow} p_{\sigma}(\ell) \text { iff } q\left(\gamma_{s}, \varepsilon_{0}+\sigma\right) \underset{s \rightarrow+\infty}{\longrightarrow} \ell
$$

Since $\gamma$ must converge to some point of $\mathbf{C}\left(\right.$ \%.(a)), we conclude from (5.16) that $\gamma_{s} \underset{s \rightarrow+\infty}{\longrightarrow} \mathbf{p}$ if we cannot find some $\sigma \in \mathbb{S}^{d-1}$ and $\ell \in \mathbb{R}$ such that (5.16) holds.

4(c). Apply Lemma 5.7 to see that if $\gamma_{s} \rightarrow p_{\sigma}(\ell)$ then $\boldsymbol{P a s t}\left(\gamma_{s}\right) \rightarrow \operatorname{Past}\left(p_{\sigma}(\ell)\right)$; the point follows from this observation.

\section{REFERENCES}

[1] A. Ancona, Théorie du potentiel sur les graphes et les variétés. École d'été de Probabilités de Saint-Flour XVIII, 1988. Lect. Notes Math. 1427 (1990) 1-112. Springer, Berlin.

[2] D. Applebaum, Compound Poisson processes and Lévy processes in groups and symmetric spaces. J. Theoret. Probab. 13 (2000) 383-425.

[3] D. Applebaum and H. Kunita, Lévy flows on manifolds and Lévy processes on Lie groups. J. Math. Kyoto Univ. 33 (1993) 1103-1123.

[4] I. Bailleul, Poisson boundary of a relativistic diffusion. Probab. Theory Related Fields 141 (2008) 283-329.

[5] P. Baldi and M. Chaleyat-Maurel, Sur l'équivalent du module de continuité des processus de diffusion, in Séminaire de Probabilités, XXI. Lect. Notes Math. 1247 (1987) 404-427. Springer, Berlin.

[6] J.K. Beem, P.E. Ehrlich and K.L. Easley, Global Lorentzian geometry, volume 202 of Monographs and Textbooks in Pure and Applied Mathematics. Marcel Dekker Inc., New York, second edition (1996).

[7] A.N. Borodin and P. Salminen, Handbook of Brownian motion - facts and formulae. Probability and its Applications. Birkhäuser Verlag, Basel. Second edition (2002).

[8] Y. Derriennic, Lois "zéro ou deux" pour les processus de Markov. Applications aux marches aléatoires. Ann. Inst. H. Poincaré Sect. B (N.S.) 12 (1976) 111-129.

[9] R.M. Dudley, Lorentz-invariant Markov processes in relativistic phase space. Ark. Mat. 6 (1966) 241-268.

[10] R.M. Dudley, Asymptotics of some relativistic Markov processes. Proc. Natl. Acad. Sci. USA 70 (1973) 3551-3555.

[11] C. Frances, Géométrie et dynamique Lorentzienne conformes. École Normale Supérieure de Lyon (2002).

[12] R. Geroch, E.H. Kronheimer, and Roger Penrose, Ideal points in space-time. Proc. Roy. Soc. Lond. Ser. A 327 (1972) $545-567$.

[13] A. Grigor'yan, Analytic and geometric background of recurrence and non-explosion of the Brownian motion on Riemannian manifolds. Bull. Amer. Math. Soc. (N.S.) 36 (1999) 135-249.

[14] Y. Guivarc'h. Une loi des grands nombres pour les groupes de Lie. In Séminaire de Probabilités, I . Exposé No. 8. Dépt. Math. Informat., Univ. Rennes, France (1976).

[15] T.R. Hurd, The projective geometry of simple cosmological models. Proc. Roy. Soc. Lond. Ser. A 397 (1985) $233-243$.

[16] N. Ikeda and S. Watanabe, Stochastic differential equations and diffusion processes. North-Holland Mathematical Library, Vol. 24. North-Holland Publishing Co., Amsterdam, second edition (1989). 
[17] F.I. Karpelevič, V.N. Tutubalin and M.G. Šur, Limit theorems for compositions of distributions in the Lobačevskiı̌ plane and space. Teor. Veroyatnost. i Primenen. 4 (1959) 432-436.

[18] M. Liao, Lévy processes in Lie groups, volume 162 of Cambridge Tracts in Mathematics. Cambridge University Press, Cambridge (2004).

[19] J. Neveu, Mathematical foundations of the calculus of probability. Translated by Amiel Feinstein. Holden-Day Inc., San Francisco, Californie (1965).

[20] B. O'Neill, Semi-Riemannian geometry. With applications to relativity, volume 103 of Pure Appl. Math. Academic Press Inc. (Harcourt Brace Jovanovich Publishers), New York (1983).

[21] R.G. Pinsky, Positive harmonic functions and diffusion, volume 45 of Cambridge Studies in Advanced Mathematics. Cambridge University Press, Cambridge (1995).

[22] J.-J. Prat, Étude asymptotique et convergence angulaire du mouvement brownien sur une variété à courbure négative. $C$. $R$. Acad. Sci. Paris Sér. A-B 280 Aiii (1975) A1539-A1542.

[23] A. Raugi, Fonctions harmoniques sur les groupes localement compacts à base dénombrable. Bull. Soc. Math. France, Mémoire 54 (1977) 5-118.

[24] A. Raugi, Périodes des fonctions harmoniques bornées. In Seminar on Probability, Rennes, 1978 (French). Exposé No. 10. Univ. Rennes, France (1978). 